\title{
Supersymmetry in Boundary Integrable Models *
}

\author{
N.P. Warner \\ Physics Department, University of Southern California \\ University Park, Los Angeles, CA 90089-0484. \\ and \\ LPTHE, Université Pierre et Marie Curie - Paris VI \\ Université Denis Diderot - Paris VII \\ Laboratoire associé No. 280 au CNRS ${ }^{\dagger}$
}

\begin{abstract}
Quantum integrable models that possess $N=2$ supersymmetry are investigated on the half-space. Conformal perturbation theory is used to identify some $N=2$ supersymmetric boundary integrable models, and the effective boundary Landau-Ginzburg formulations are constructed. It is found that $N=2$ supersymmetry largely determines the boundary action in terms of the bulk, and in particular, the boundary bosonic potential is $|W|^{2}$, where $W$ is the bulk superpotential. Supersymmetry is also investigated using the affine quantum group symmetry of exact scattering matrices, and the affine quantum group symmetry of boundary reflection matrices is analyzed both for supersymmetric and more general models. Some $N=2$ supersymmetry preserving boundary reflection matrices are given, and their connection with the boundary Landau-Ginzburg actions is discussed.
\end{abstract}

USC-95/014, hep-th/9506064

June 1995

* Work supported in part by funds provided by the DOE under grant No. DE-FG0384ER40168.

$\dagger$ Boite 126, Tour 16, $1^{\text {er }}$ étage, 4 place Jussieu, F-75252 Paris CEDEX 05, FRANCE. 


\section{Introduction}

The study of conformal and quantum integrable models in two-dimensional systems with a boundary is not only an intrinsically interesting subject, but also provides yet another avenue by which one can relate integrable models to physically relevant systems and, in particular to situations that are of interest in three or four dimensional field theory. The basic two-dimensional boundary model consists of a system defined upon the half-line $(x<0)$ with a spatial boundary at $x=0$. The field theory in the bulk (i.e. for $x<0)$ can be either conformal or quantum integrable, while the boundary conditions can be simple (free or fixed), or non-trivial, involving a mass scale and possibly non-linear boundary couplings and dynamics. For the boundary model to be integrable (i.e. possess higher spin conserved charges), there are stringent constraints upon the bulk and boundary sectors (for example, see [1,2, 2, 3, 团).

Probably the most famous physical example of a boundary integrable model occurs in the Kondo problem, where the $(1+1)$-dimensional field theory is the effective field theory of $s$-wave scattering of electrons off a magnetic spin impurity. The bulk theory is massless (conformal) while the boundary has a mass scale (the Kondo temperature). Such impurity problems, in which one concentrates on $s$-wave scattering off some isolated object at the origin, generically provide interesting $(1+1)$-dimensional boundary field theories. Some of these may be quantum integrable, or may have physically useful approximations that are integrable. Another example of this is the Callan-Rubakov effect [5], where scattering of fermions off a monopole is reduced to the study of free fermions on the half-line.

There are also intrinsically $(1+1)$-dimensional boundary quantum integrable systems of experimental relevance: There is a significant body of evidence to support a Luttinger liquid (Thirring) model for the edge states of electrons in the fractional quantum Hall effect [6], and the boundary sine-Gordon model thus provides an accurate description of the conductance though a point contact [7].

In this paper I will consider supersymmetry in boundary integrable models. The idea is to start with an $N=2$ superconformal model on the plane, and to see to what extent supersymmetry can be preserved once one passes to the half-space and perturbs with relevant boundary, and possibly bulk, operators that lead to a quantum integrable theory. There are several motivations for considering this issue. First, if one has $N=2$ supersymmetry on the plane or cylinder, then one can obtain quite a number of exact quantum properties of such models via semi-classical analysis: One can determine the 
vacuum structure and soliton mass ratios from an effective Landau-Ginzburg formulation [8,9.10]; one can compute some partition functions from the elliptic genus [11.12], and get some exact scaling functions from the generalized index [13]. It is thus natural to investigate whether one will be able to generalize these sorts of results to the half-space. At a more formal level, the supersymmetric models, and their topological counterparts, provide the simplest examples of Coulomb gas methods, along with the associated action of the affine quantum group on the soliton spectrum and $S$-matrices. Once again, one would like to know how much of these structures survive for quantum integrable models on the half-space. Finally, from the point of view of higher dimensional field theories, if one considers monopoles or strings in a supersymmetric field theory, and treats them as impurity problems, then the result will be supersymmetric $(1+1)$-dimensional boundary field theories. In some limit these will be superconformal models, and if one is lucky, in some approximation where mass scales are re-introduced, one might get an integrable field theory. It is thus important to study supersymmetry in boundary field theories, and investigate to what extent the supersymmetry is broken by the presence of the boundary, and which of these models are quantum integrable.

I will start in section 2 by reviewing some pertinent facts about conformal field theory and extended chiral algebras on the half-space. In section 3, I review the conditions under which boundary and bulk perturbations lead to quantum integrable models, and section 4 contains an analysis of the $N=2$ supersymmetric boundary integrable models that can be obtained from perturbations of the $N=2$ superconformal minimal series. In particular, it is shown how families of such models can be obtained from the bulk perturbations that lead to superpotentials that are Chebyshev polynomials. The second major thread of this work is introduced in section 5, where exact scattering matrices and their affine quantum group symmetries are employed in an examination of the supersymmetry of one of the simplest supersymmetric boundary integrable models. In section 6 , I construct some exact boundary reflection matrices that have both free parameters and preserve $N=2$ supersymmetry. Finally, section 7 gives the construction of the effective Landau-Ginzburg theories for the models described in the earlier sections, and in particular it is found that if the bulk superpotential is $W(\phi)$, then the boundary superpotential, $V$, satifies $\frac{\partial V}{\partial \phi}=W$, and hence the boundary bosonic potential is $|W|^{2}$. 


\section{Conformal Systems with a Boundary}

Consider a conformal field theory on the complete complex plane, and suppose that the theory is symmetric between the left-moving and right-moving sectors. In particular, this means that every left-moving operator, $\mathcal{O}(z)$, in the chiral algebra $\mathcal{A}$, has a right-moving counterpart, $\widetilde{\mathcal{O}}(\bar{z})$, in the chiral algebra $\widetilde{\mathcal{A}} \equiv \mathcal{A}$. As is familiar in open string theory, the introduction of a boundary requires that the left-movers and right-movers be locked together. As a result, the two chiral algebras, $\mathcal{A}$ and $\widetilde{\mathcal{A}}$, become identified, producing a single copy, $\hat{\mathcal{A}}$, of $\mathcal{A}$ in the system with the boundary. Perhaps the simplest way to think of this is as a generalized method of images: That is, $\mathcal{A}$-preserving boundary conditions require [14]:

$$
\left.\mathcal{O}(z)\right|_{x=0}=\left.\widetilde{\mathcal{O}}(-\bar{z})\right|_{x=0} .
$$

One can then think of $\widetilde{\mathcal{O}}(-\bar{z})$ as the analytic continuation of $\mathcal{O}(z)$ into $x=\mathcal{R} e(z)>0$.

To define the conserved charges, $\mathcal{O}_{n}$, it is convenient to introduce semi-circular contours $C_{L}$ and $C_{R}$, centered on the origin, $O$. These contours are mirror images of each other through the $y$-axis, and have bases that run along the $y$-axis (see Fig.1). Let $C$ be the complete circular contour about $O$, made up of the semi-circular $\operatorname{arcs}$ of $C_{L}$ and $C_{R}$. Then define:

$$
\begin{aligned}
\mathcal{O}_{n} & =\frac{1}{2 \pi i} \oint_{C_{L}} z^{n+p-1} \mathcal{O}(z) d z-\frac{1}{2 \pi i} \oint_{C_{R}}(-\bar{z})^{n+p-1} \widetilde{\mathcal{O}}(-\bar{z}) d(-\bar{z}) \\
& =\frac{1}{2 \pi i} \oint_{C} z^{n+p-1} \mathcal{O}(z) d z
\end{aligned}
$$

where $p$ is the conformal weight of $\mathcal{O}(z)$.

For $N=2$ superconformal boundary conditions one requires:

$$
\begin{aligned}
\left.J(z)\right|_{x=0} & =\left.\widetilde{J}(-\bar{z})\right|_{x=0}, \\
\left.T(z)\right|_{x=0} & =\left.\widetilde{T}(-\bar{z})\right|_{x=0}, \\
\left.G^{ \pm}(z)\right|_{x=0} & =\left.\widetilde{G}^{ \pm}(-\bar{z})\right|_{x=0} .
\end{aligned}
$$

The result is a single $N=2$ superconformal algebra on the half-space. The choice of pairing $G^{ \pm}(z)$ with $\widetilde{G}^{ \pm}(-\bar{z})$ or with $\widetilde{G}^{\mp}(-\bar{z})$ is a matter of convention, but changing this pairing will introduce a relative negative sign in the pairing of $J(z)$ and $\widetilde{J}(z)$. 


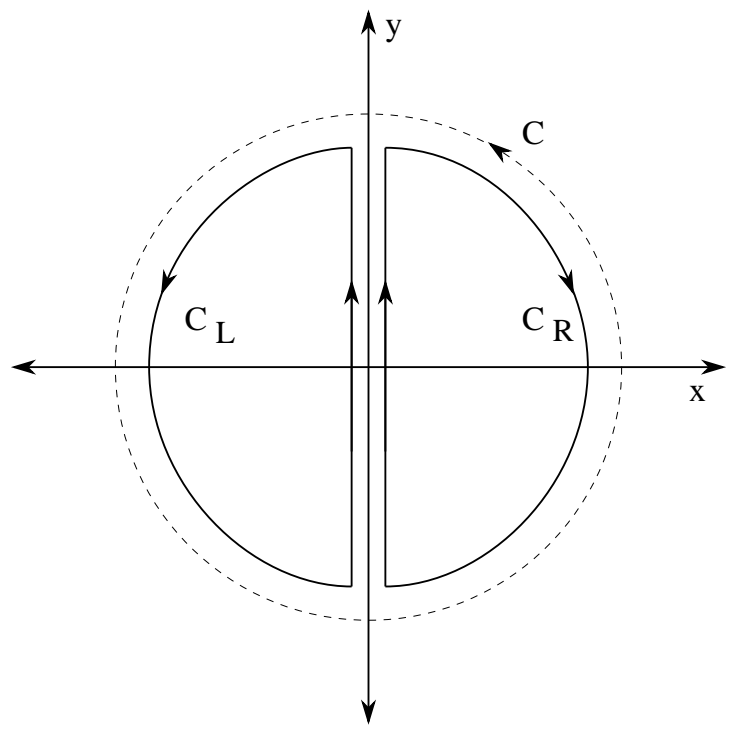

Fig.1: The contours for defining modes of operators on the half space.

One should also note that the boundary conditions (2.1) do not completely determine the boundary conditions for the individual primary fields in the theory, but merely guarantee that the theory on the half space is $\mathcal{A}$-conformal (i.e. conformal with chiral algebra $\mathcal{A}) 1$.

\section{Boundary integrable models}

The initial focus of this paper will be insertions of boundary perturbations of the form:

$$
A_{b d r y}=\mu \int_{-\infty}^{\infty} \chi(t) d t
$$

where $\chi(t)$ is some operator defined upon the boundary at $x=0$. To be relevant (or marginal), the operator $\chi$ must have dimension less than (or equal to) one. For a relevant boundary operator, the coupling constant, $\mu$, introduces a boundary mass scale.

I will also consider bulk perturbations of the form:

$$
\Delta A_{b u l k}=g \int_{\mathcal{R} e(z)<0} \psi(z, \bar{z}) d^{2} z
$$

where $g$ is a coupling constant, and $\psi(z, \bar{z})$ is generically a sum of products of holomorphic and anti-holomorphic operators: $\psi(z, \bar{z})=\sum_{j} \psi_{j}(z) \tilde{\psi}_{j}(\bar{z})$.

1 One could also imagine imposing boundary conditions that violate (2.1) for some of the generators of the chiral algebra. Such boundary conditions can reduce, or at least modify the structure of the chiral algebra on the half-space. A familiar, though simple, version of this is the choice of Neveu-Schwarz or Ramond sectors in the open string. 


\subsection{Conformal perturbation theory}

To first order in conformal perturbation theory, the crucial issue in whether a boundary perturbation leads to an integrable boundary field theory is precisely what representation the boundary operator has with respect to the underlying chiral algebra on the halfspace, and not how this operator may, or may not, be obtained from the limit of bulk operators. This is because one tests a putative conserved current, $\mathcal{O}(z)$, in the presence of perturbation, $\psi(z, \bar{z})=\psi(z) \tilde{\psi}(\bar{z})$, by computing corrections to the conformal Ward indentity $\bar{\partial} \mathcal{O}(z)=0$. This current will remain conserved if the corrections to this Ward identity have the form $L_{-1} \mathcal{X}$, for some operator $\mathcal{X}$. At first order in perturbation theory, this will be true if and only if the coefficient of the simple pole in the operator product $\mathcal{O}(z) \psi(w)$ is $L_{-1}$ of some operator [15]. The result of this operator product computation depends upon the operator $\mathcal{O}(z)$ and the representation generated by $\psi(w)$ under the action of the chiral algebra (in particular, the null vectors are usually crucial). If one finds that the coefficient of the simple pole is indeed $L_{-1}$ of something for a particular bulk operator, $\psi$, then this is a statement of representation theory, and must also be true for a boundary operator in the same representation of the chiral algebra of the theory on the half-space. However, for the boundary theory, the operator $L_{-1}$ generates translations that

preserve the boundary, that is, $L_{-1}=\frac{\partial}{\partial t}$. Therefore, the Ward identity $\bar{\partial} \mathcal{O}(z)=0$ gets corrected by $\frac{\partial}{\partial t}$ of some boundary operator. Thus one obtains the boundary corrections to a conserved charge in the boundary integrable model in exactly the same manner that one computes the bulk corrections to a conserved current in a bulk integrable model. This means that, at least to first order in perturbation theory, if one has a bulk perturbation that leads to a quantum integrable field theory, then one can make a boundary integrable field theory provided that one can construct a well-defined boundary operator in the same representation of the chiral algebra. The foregoing argument is basically a rephrasing of the argument given in [1]. The last proviso about actually constructing boundary operators in the requisite representations is where the subtleties lie [14].

To illustrate these issues it is instructive to consider the Ising model, for which there are two known bulk integrable models. The simplest is obtained from the energy perturbation of the conformal model, where the perturbing operator belongs to the $\Phi_{1,3}$ representation of the Virasoro algebra. This perturbation corresponds to giving a mass to the underlying free fermion. Following the logic above, it follows that a boundary operator in the $\Phi_{1,3}$ representation must lead to an integrable model. This is indeed true [1], but 
the correponding boundary operator is the boundary magnetic perturbation [14,1]. The easiest way to see this is to consider the Ising model with free boundary conditions, and look at what happens when one takes a spin operator, $\sigma(z, \bar{z})$, to the boundary. At the boundary, the holomorphic and anti-holomorphic parts of the spin field fuse into a single representation of the Virasoro algebra. (Put differently, the holomorphic part of the spin field meets its mirror image in the boundary.) The net result is to get the fusion product of two spin fields, which generates the identity and the energy operator $\left(\Phi_{1,3}\right)$. The fusion to the identity disappears because one started with free boundary conditions, and so one gets the required energy operator at the boundary [14]. More generally, by making a choice of conformal boundary conditions, one immediately restricts the representations of operators that can be constructed on the boundary.

It is also instructive to consider the boundary analogue of the other integrable perturbation of the Ising model. The bulk perturbation corresponds to the magnetic operator, or the $\Phi_{1,2}$ representation of the Virasoro algebra [15]. One cannot obtain a boundary operator in this representation of the Virasoro algebra by taking a limit of bulk operators. Indeed, if one starts with the standard conformal boundary conditions (free or fixed) there is simply no such operator in the boundary spectrum [14]. There are, however, boundary condition changing operators in precisely the right representation [14], and so it is tantalizing to suggest that a "perturbation" by such operators could yield the boundary analogue of the bulk magnetic perturbation that leads to a quantum integrable model with an $E_{8}$ structure [15]. The immediate problem that one runs into is that one needs answer the question: what is the conformal fixed point about which one is perturbing? Alternatively, what is the U.V. fixed point of such a theory where the boundary coupling vanishes? It is certainly not fixed or free boundary conditions for the reasons mentioned above. One might hope to find some more general conformal boundary condition. Indeed, if one attempts a vague interpretation of what the boundary condition changing operator in the $\Phi_{1,2}$ representation does, then it seems to want to make a U.V. fixed point that would look like an average over a boundary magnetic field that takes values $\pm \infty$ or 0 for random intervals of random length. It is, as yet, unclear to me whether this idea can be turned into a more precise or meaningful proposal.

The essential conclusion is that perturbations of conformal field theories fall into two classes, those that are in representations of the chiral algebra that can be obtained in the boundary spectrum of some conformal boundary condition, and those that are not. If the bulk perturbation leads to a bulk integrable model, then the former class of "good" 
perturbations lead to boundary integrable models, while the latter class are "problematic" perturbations whose naive boundary analogues may not exist.

One should bear in mind that the arguments above are only to first order in conformal perturbation theory. If the perturbing operators are very relevant (i.e. of low dimension) then simple dimensional arguments can be used to show that first order perturbation theory is sufficient to establish the existence of higher spin conserved charges to all orders [15]. On the other hand, if the perturbing operators are nearly marginal, then there could be higher order corrections to the conserved charges. For bulk perturbations, we know from free field formulations, and related Toda theories, that even though there might be such corrections, they do not spoil quantum integrability [16]. Given the argument above, and the close parallel between bulk and boundary operators, it seems very plausible to expect the same to be true for boundary perturbations $⿴$ 日.

Suppose now that one makes simultaneous and independent perturbations on the boundary and in the bulk using (3.1) and (3.2), where $\chi(t)$ and $\psi(z)$ are in the same representations of the chiral algebra. It follows from the first order conformal perturbation theory that if a current $\mathcal{O}(z)$ leads to a conserved charge in the presence of the bulk perturbation, then it also does so in the presence of the independent bulk and boundary perturbations. It is, of course, quite possible that the bulk and boundary perturbations could interact at higher orders in perturbation theory and thus further constrain, or perhaps even destroy, quantum integrability. Once again, this seems unlikely, and if one considers perturbing operators of low enough dimension then it cannot happen.

\subsection{Spin-1 currents and topological charges}

There are two important exceptions to the standard first-order conformal perturbation theory argument presented above. The first is trivial, and concerns spin-1 currents. The holomorphic and anti-holomorphic parts, $J(z)$ and $\widetilde{J}(\bar{z})$, of a spin-1 current in a conformal model are separately conserved. If one makes a bulk perturbation, then $J(z)$ and $\widetilde{J}(\bar{z})$ give rise to a single conserved $U(1)$ current if and only if the perturbing operator is neutral with respect to some linear combination of the associated left-moving and right-moving charges. While this is obvious, the computation in terms of conformal perturbation theory is substantially different from the argument above, and most importantly, even though a

2 There are however some complications if one attempts to use Toda and free field arguments directly for boundary field theories. This is discussed below. 
bulk perturbation can preserve a $U(1)$ charge, the corresponding boundary perturbation may well destroy it (even at first order).

The second exception is a little more subtle, and arises whenever there are bulk topological charges. Such topological charges appear in massive theories with extended supersymmetry. Even at first order in perturbation theory one finds that a superconformal algebra will receive corrections that generate first order corrections involving total derivatives of bosonic fields. These corrections therefore contribute bosonic terms to the algebra, and these depend upon the boundary conditions. In infinite systems the boundary conditions are usually fixed, and these boundary terms are referred to as topological charges (for example, see [8,9,10,17]). In the semi-infinite system these topological charges will only be conserved if the bosonic boundary terms are fixed. A Priori this means that the superalgebra will be, to some extent, broken unless some form of Dirichlet boundary conditions are imposed. Therefore, even though first order perturbation theory indicates no problems for each supercharge individually, the supersymmetry can be broken even at first order. I suspect that this can only occur in systems that have conserved currents of fractional spin, whereas, in the more conventional systems with commuting integer spin charges, the issue of such topological charges will probably not arise.

For supersymmetric theories in semi-infinite systems, there several ways in which one can ameliorate, or even avoid, the problems caused by topological charges, and thereby preserve the supersymmetries. One can:

(i) Enforce Dirichlet boundary conditions.

(ii) Keep the bulk conformally invariant, and only perturb on the boundary. There are no topological charges in a bulk superconformal algebra.

(iii) Find combinations of supercharges such that the topological charges do not effectively contribute to the commutator.

(iv) Try to compensate for the topological charge terms by using boundary degrees of freedom, and making re-definitions of the boundary supersymmetry and hamiltonian. To illustrate possibility (iv), suppose that a bulk topological charge term appears in the square of a supersymmetry generator and gives rise to a boundary topological term $\mathcal{X}$. One can cancel this term by introducing boundary fermions $b$ and $b^{\dagger}$, with $\left\{b, b^{\dagger}\right\}=1$, and then adding boundary correction of $b-\mathcal{X} b^{\dagger}$ to the supercharge. Indeed, it will be shown in section 7 that it is precisely this mechanism that enables one to construct $N=2$ supersymmetry boundary integrable models with non-trivial boundary interactions. 


\subsection{An Aside: Toda models}

In the presence of a boundary, the simply-laced classical Toda models, of rank more than one, are more tightly constrained than the perturbed conformal models: In the Toda models, the boundary couplings are fixed in terms of the bulk couplings [3,4]. What is also surprising is that the non-simply-laced models are not so tightly constrained [3]. Thus the Toda models all give rise to quantum integrable boundary theories, but some have the boundary and bulk mass scales locked together while others do not.

A correspondence between Toda models, with imaginary coupling constants, and perturbed conformal field theories can be made by viewing all but one, or two, of the Toda potential terms as defining a Coulomb gas prescription, and then the remaining potential terms are re-interpreted as free field forms of the relevant perturbations of the conformal model [18,16]. This correspondence has proved a very valuable tool in bulk theories, and one would therefore hope to be able to extend it to boundary theories. However, conformal perturbation theory produces no constraints between bulk and boundary mass scales.

There are thus several possible ways in which one might account for this lack of constraint. First, it is possible that the constraint appears at higher orders in conformal perturbation theory. However, the exact $S$-matrices and effective actions suggest otherwise; and in some instances one might be able to use dimensional analysis to exclude the possibility of such higher order corrections. Another possibility is that the introduction of massive, non-linear boundary conditions at the level of the Toda action could conceivably destroy, or interfere with the Coulomb gas interpretation, particularly if the boundary terms modify the scaling properties of what one hopes to interpret as the screening charges. A third possibility, which is supported by later results in this paper, is that to properly define the boundary action so as to enable a Coulomb gas interpretation, one may need to introduce new boundary degrees of freedom and specify their dynamics. Without these degrees of freedom, the Coulomb gas methods might fail, and the correspondence with conformal perturbation theory may be be lost.

There is also an important observation that could provide some explanation of the apparent difference between boundary Toda theories and conformal perturbation theory in systems with boundary. Suppose that a bulk perturbation of a conformal model were in a representation of the chiral algebra that was not in the boundary spectrum of any conformal boundary conditions for the bulk model - just like the $\Phi_{1,2}$ perturbation of the conformal Ising model. Then it will be impossible to find a boundary perturbation 
of the corresponding Toda model (with imaginary coupling) such that the model remains quantum integrable and possesses a limit in which the bulk becomes massless, while the boundary remains massive. It follows that there will be families of bulk perturbations that lead to bulk integrable models, but that do not have boundary analogues for which there is a massless bulk limit. It is natural to conjecture that the models in which the bulk and boundary mass scales are fixed in terms of one another are precisely in this class. Certainly the perturbations of Ising fit this pattern.

If this conjecture is correct, it is then intriguing that one is, in fact, able to construct a boundary Toda model with non-trivial boundary interaction in the first place, since this would imply that conformally forbidden boundary interactions will become permissible so long as they are always combined with suitable massive bulk interactions.

It is obviously valuable to check this conjecture against the known examples. It would also be useful to see whether there is a characterization of this boundary spectrum statement that can be made entirely in terms of a Toda field theory. Since this issue is not central to this paper, I will not pursue it further here.

\section{Some supersymmetry preserving boundary perturbations}

I will, for simplicity, focus on the $N=2$ superconformal minimal models with $A$ type modular invariants, and whose central charge is $c=3 k /(k+2)$. The relevant bulk perturbations of these models that lead to $N=2$ supersymmetric integrable field theories are well known [9, 19,20]. There are three distinct such perturbations, but the one of importance here is the Chebyshev perturbation. That is, one perturbs the bulk using

$$
\lambda \int G_{-\frac{1}{2}}^{-} \widetilde{G}_{-\frac{1}{2}}^{-} \phi_{k}^{+}(z, \bar{z}) d^{2} z+\bar{\lambda} \int G_{-\frac{1}{2}}^{+} \widetilde{G}_{-\frac{1}{2}}^{+} \phi_{k}^{-}(z, \bar{z}) d^{2} z
$$

where $\lambda$ is a coupling constant, $\phi_{k}^{+}$is the chiral primary field of charge $q=\tilde{q}=\frac{k}{k+2}$ and conformal weight $h=\tilde{h}=\frac{k}{2(k+2)}$, and $\phi_{k}^{-}$is the anti-chiral conjugate of $\phi_{k}^{+}$. This perturbation leads to a massive $N=2$ supersymmetric integrable model whose effective Landau-Ginzburg potential is the Chebyshev polynomial of degree $(k+2)$ (see, for example [21]). This may be thought of as the $N=2$ superconformal analogue of the energy perturbation of the ordinary minimal series.

Suppose that $\psi^{ \pm}(t)$ are boundary operators that transform in exactly the same representations as the holomorphic operators $G_{-\frac{1}{2}}^{\mp} \phi_{k}^{ \pm}(z)$ under the action of the $N=2$ 
superconformal algebra. In particular this implies that the operators $\psi^{ \pm}(t)$ may be written

$$
\psi^{ \pm}(t)=G_{-\frac{1}{2}}^{\mp}\left(\hat{\phi}_{k}^{ \pm}(t)\right)
$$

where $G_{-\frac{1}{2}}^{\mp}$ are the supercharges of the boundary theory, and $\hat{\phi}^{ \pm}(t)$ are boundary operators in the same $N=2$ superconformal representation as $\phi^{ \pm}(z)$ and $\phi^{ \pm}(\bar{z})$.

In order to use $\psi^{ \pm}(t)$ in a boundary perturbation it is necessary to show that these operators are in the boundary spectrum of some conformally invariant boundary condition. This is straightforward, and is completely parallel with the analogous situation in the Ising model. Let $\phi_{1}^{+}(z, \bar{z})$ be the most relevant chiral primary field (with $q=\tilde{q}=\frac{1}{k+2}$ and conformal weight $\left.h=\tilde{h}=\frac{1}{2(k+2)}\right)$. This field is the basic order parameter of the massive, bulk integrable model [22,9]. If we send this bulk field to the boundary, the result is an operator in the fusion product of $\phi_{1}^{+}(z)$ with itself. There are two fields in this fusion product: (i) $\phi_{2}^{+}$, the chiral primary with $q=\frac{2}{k+2}$ and conformal weight $h=\frac{2}{2(k+2)}$, and (ii) the field $G_{-\frac{1}{2}}^{+} \hat{\phi}_{k}^{-}$, with $q=1-\frac{k}{k+2}=\frac{2}{k+2}$ and conformal weight $h=\frac{1}{2}+\frac{k}{2(k+2)}=\frac{k+1}{k+2}$. If one starts with the $N=2$ superconformal model with free boundary conditions in the Landau-Ginzburg formulation, then the expectation value of $\phi_{2}^{+}=\left(\phi_{1}^{+}\right)^{2}$ will vanish at the boundary, and one will get the sub-leading operator $\psi^{-}(t)=G_{-\frac{1}{2}}^{+} \hat{\phi}_{k}^{-}(t)$. Similarly, one obtains $\psi^{+}(t)=G_{-\frac{1}{2}}^{-} \hat{\phi}_{k}^{+}(t)$ by sending the anti-chiral conjugate, $\phi_{1}^{-}(z, \bar{z})$, of $\phi_{1}^{+}(z, \bar{z})$, to the boundary.

Conformal perturbation theory can now be invoked to show that (at least to first order) one can obtain a boundary integrable model from a simultaneous bulk perturbation using (4.1) and boundary perturbation using (3.1) with:

$$
\chi(t)=\nu \psi^{+}(t)+\bar{\nu} \psi^{-}(t)
$$

The constant $\nu$ is a complex phase, with complex conjugate $\bar{\nu}$. If there is no bulk perturbation then one can absorb this phase $\nu$ into a re-definition of $\phi_{k}^{ \pm}$. If there is a bulk perturbation, then this freedom of re-definition can be used to adjust the phase of $\lambda$ (in (4.1)) or the phase $\nu$. Thus there are three independent couplings: the bulk mass scale, determined by $|\lambda|$, the boundary mass scale, determined by $\mu$ in (3.1), and the relative phase between $\lambda / \bar{\lambda}$ and $\nu / \bar{\nu}$.

It is well known that the bulk perturbation (4.1), in the infinite domain, preserves all of the supersymmetry [9,23,24], and provides one with a massive $N=2$ supersymmetric model. There are two ways of seeing the latter: One can either explicitly verify the result 
using conformal perturbation theory, or, more generally, one can appeal to the theory of supersymmetric actions, which states that perturbations that involve only top components of superfields (as does (4.1)) preserve the supersymmetry. The same arguments can also be naively applied to the boundary perturbation (4.3), with similar conclusions. However, the massive bulk model has topological charges, and so the supersymmetry variation of the bulk action generates boundary terms via total derivatives. As described earlier, unless one chooses Dirichlet boundary conditions, or make the bulk massless, one is going to have to be very careful with the boundary interaction if one is going to preserve $N=2$ supersymmetry. I will return to this issue later, but first I think it useful to present a simple and fairly complete example, and re-examine the whole issue of supersymmetry using exact scattering matrices.

\section{The sine-Gordon model}

The simplest example of an $N=2$ superconformal minimal model is the $k=1, c=1$ minimal model that can be realized by a single free boson compactified at the "supersymmetric" radius. The superconformal generators can be written in terms of the holomorphic part of a canonically normalized boson, $\varphi(z)$, as:

$$
T(z)=-\frac{1}{2}(\partial \varphi(z))^{2} ; \quad J(z)=\frac{i}{\sqrt{3}} \partial \varphi(z) ; \quad G^{ \pm}(z)=e^{ \pm i \sqrt{3} \varphi(z)} .
$$

If $\tilde{\varphi}(\bar{z})$ denotes the anti-holomorphic part of the boson, then the $N=2$ superconformal boundary condition implies that $\left.\varphi(z)\right|_{x=0}=\left.\tilde{\varphi}(-\bar{z})\right|_{x=0}$. The order parameter, and its conjugate, are given by $\phi_{1}^{ \pm}(z, \bar{z})=e^{ \pm \frac{i}{\sqrt{3}}(\varphi(z)+\varphi(\bar{z}))}$, and as $x \rightarrow 0$ this becomes $\left.e^{ \pm \frac{2 i}{\sqrt{3}} \varphi(z)}\right|_{x=0}=$ $\left.G_{-\frac{1}{2}}^{\mp} \phi_{1}^{ \pm}(z)\right|_{x=0}$. The bulk integrable perturbations are $G_{-\frac{1}{2}}^{\mp} \widetilde{G}_{-\frac{1}{2}}^{\mp} \phi_{1}^{ \pm}=e^{\mp \frac{2 i}{\sqrt{3}}(\varphi(z)+\varphi(\bar{z}))}$. We are thus dealing with the boundary sine-Gordon theory described of [1], with action:

$$
\begin{gathered}
\int_{-\infty}^{\infty} \int_{x<0}\left(\frac{1}{2}\left(\partial_{t} \Phi\right)^{2}-\frac{1}{2}\left(\partial_{x} \Phi\right)^{2}\right)-\frac{M}{\beta}[\cos (\beta \Phi)-1] d x d t \\
-\frac{2 m}{\beta} \int_{-\infty}^{\infty} \cos \frac{\beta}{2}\left(\Phi-\Phi_{0}\right) d t
\end{gathered}
$$

From this one finds that $\Phi$ satisfies the usual sine-Gordon equation with boundary condition:

$$
\left.\partial \Phi\right|_{x=0}=\left.m \sin \frac{\beta}{2}\left(\Phi-\Phi_{0}\right)\right|_{x=0} .
$$

The boson, $\Phi$, has the standard normalization of sine-Gordon theory, for which the supersymmetry point corresponds to $\beta^{2}=\frac{16 \pi}{3}$. The parameters $M, m$ and $\Phi_{0}$ coincide with the three parameters described in the last section. It is known that this model is indeed integrable for arbitrary choices of $M, m$ and $\Phi_{0}$, and so the boundary and bulk perturbations are simultaneously and independently integrable. 


\subsection{Supersymmetry}

The bulk $S$-matrix for the sine-Gordon model has been known for a long time [25]. At the supersymmetry point one is in the repulsive regime, and so the fundamental spectrum consists of the two kinks: a soliton and an anti-soliton; there are no breathers. The soliton and anti-soliton have fermion numbers $+\frac{1}{2}$ and $-\frac{1}{2}$ respectively, and they form a two-dimensional supermultiplet [9, 17,23]. As explained in [17, the bulk $S$-matrix commutes with the generators of the affine quantum group in the principal gradation. At the supersymmetry point, the affine quantum group generators have spin $\frac{1}{2}$ and are precisely the four generators of the massive superalgebra. On the doublet consisting of the soliton and anti-soliton, the action of these supersymmetry generators is given by:

$$
\mathcal{Q}_{ \pm}(\theta)=C e^{+\frac{\theta}{2}} E_{ \pm} q^{ \pm \frac{H}{2}} ; \quad \widetilde{\mathcal{Q}}_{ \pm}(\theta)=C e^{-\frac{\theta}{2}} E_{ \pm} q^{\mp \frac{H}{2}}
$$

where $H, E_{+}$and $E_{-}$are the $2 \times 2$ matrices generating the $S U(2)$ algebra, with $\left[H, E_{ \pm}\right]=$ $\pm 2 E_{ \pm}$and $\left[E_{+}, E_{-}\right]=H$. (I will use the notation and conventions of [17].) In (5.4), the parameter, $\theta$, is the rapidity of the kink that is being acted upon, and $C$ is a constant that satisfies $C^{2}=\frac{|\lambda|}{2 \pi i}\left(1-q^{2}\right)$. The parameter, $q$, is the usual parameter of the quantum group, and is given by $q=-e^{i \pi\left(1-8 \pi / \beta^{2}\right)}$, which reduces to $q=i$ at the supersymmetry point.

To extend the action of the quantum group onto multi-kink states one introduces the co-product $\Delta$. At the supersymmetry point, the co-product introduces phases appropriate to "commuting" fermionic generators through kinks of fermion number $\pm \frac{1}{2}$. The fact that the supersymmetries commute with the $S$ matrix is then written:

$$
\left[\mathcal{S}(\theta), \Delta\left(\mathcal{Q}_{ \pm}(\theta)\right)\right]=\left[\mathcal{S}(\theta), \Delta\left(\widetilde{\mathcal{Q}}_{ \pm}(\theta)\right)\right]=0
$$

The algebra of the bulk supercharges is:

$$
\begin{aligned}
& \left\{\mathcal{Q}_{ \pm}, \widetilde{\mathcal{Q}}_{ \pm}\right\}=0 ; \quad\left\{\mathcal{Q}_{ \pm}, \widetilde{\mathcal{Q}}_{\mp}\right\}=\frac{|\lambda|}{2 \pi i}\left(1-q^{ \pm 2 \mathcal{T}}\right) \\
& \left\{\mathcal{Q}_{+}, \mathcal{Q}_{-}\right\}=\frac{|\lambda|}{2 \pi i} q^{-1}\left(1-q^{2}\right) \mathcal{P}_{+} ; \quad\left\{\widetilde{\mathcal{Q}}_{+}, \widetilde{\mathcal{Q}}_{-}\right\}=\frac{|\lambda|}{2 \pi i} q\left(1-q^{2}\right) \mathcal{P}_{-}
\end{aligned}
$$

where $q=i$. The operators $\frac{|\lambda|}{2 \pi i} \mathcal{P}_{ \pm}$are the light-cone components of the momentum, and $\mathcal{T}=H$, is the generator of the $U(1)$ in $U_{q}(S U(2))$, and is given by the bulk topological charge:

$$
\mathcal{T}=H=\frac{\beta}{2 \pi} \int_{-\infty}^{\infty} \partial_{x} \Phi d x .
$$

For a bulk superconformal model, the coupling, $\lambda$, and momenta, $\mathcal{P}_{ \pm}$, are scaled in such a manner that $\frac{|\lambda|}{2 \pi i} \mathcal{P}_{ \pm}$remains finite, while the topological terms: $\frac{|\lambda|}{2 \pi i}\left(1-q^{ \pm 2 \mathcal{T}}\right)$ scale to zero. As a result, there are no topological terms in the algebra in the bulk massless limit. 


\subsection{Incorporating the boundary}

For the boundary theory (5.2), conformal perturbation theory leads one to expect that two supersymmetry generators will survive. There are two possibilities for the pair of surviving supercharges:

$$
\text { either } \quad \widehat{\mathcal{Q}}_{ \pm}=\mathcal{Q}_{ \pm}+e^{ \pm i \omega} q^{-1} \widetilde{\mathcal{Q}}_{ \pm} \quad \text { or } \quad \widehat{\mathcal{Q}}_{ \pm}^{\prime}=\mathcal{Q}_{ \pm}+e^{ \pm i \omega} q^{-1} \widetilde{\mathcal{Q}}_{\mp}
$$

The phase, $\omega$, is an arbitrary constant. The choice between $\widehat{\mathcal{Q}}_{ \pm}$and $\widehat{\mathcal{Q}}_{ \pm}^{\prime}$ depends upon the boundary condition for the $U(1)$ current. The algebras are slightly different:

$$
\begin{aligned}
& \left\{\widehat{\mathcal{Q}}_{ \pm}, \widehat{\mathcal{Q}}_{ \pm}\right\}=0, \\
& \begin{array}{l}
\left\{\widehat{\mathcal{Q}}_{ \pm}, \widehat{\mathcal{Q}}_{\mp}\right\}=\frac{|\lambda|}{2 \pi i} q^{-1}\left(1-q^{2}\right)\left[\left(\mathcal{P}_{+}+\mathcal{P}_{-}\right)+\right. \\
\left.\frac{1}{\left(1-q^{2}\right)}\left(2 \cos \omega-e^{-i \omega} q^{+2 \mathcal{T}}-e^{i \omega} q^{-2 \mathcal{T}}\right)\right]
\end{array}
\end{aligned}
$$

whereas, one has:

$$
\begin{aligned}
& \left\{\widehat{\mathcal{Q}}_{ \pm}^{\prime}, \widehat{\mathcal{Q}}_{ \pm}^{\prime}\right\}=2 e^{ \pm i \omega} q^{-1} \frac{|\lambda|}{2 \pi i}\left(1-q^{ \pm 2 \mathcal{T}}\right) \\
& \left\{\widehat{\mathcal{Q}}_{ \pm}^{\prime}, \widehat{\mathcal{Q}}_{\mp}^{\prime}\right\}=q^{-1} \frac{|\lambda|}{2 \pi i}\left(1-q^{2}\right)\left(\mathcal{P}_{+}+\mathcal{P}_{-}\right)
\end{aligned}
$$

Note that only the combination, $\mathcal{P}_{+}+\mathcal{P}_{-}$, appears in these algebras. Indeed, the relative phases in (5.8) were chosen so as to produce this combination. This is because the boundary conserves energy, but not momentum, and the operator $\mathcal{P}_{+}+\mathcal{P}_{-}$is the dimensionless energy operator: $E / M_{0}$, where $M_{0}$ is the soliton mass.

While both the superalgebras, (5.9) and (5.10), have two generators, they are different. In the first, one cannot separate the topological terms from the energy operator, whereas one can in the second algebra. One can also see this distinction is not an artefact of basis choice by looking at unitarity bounds on the energy that can be deduced from the superalgebra.

To compute precisely what is happening with supersymmetry in this model one can use the exact boundary reflection matrix of $\mathbb{1}$. That is, if one assumes that there is no boundary structure, one can use boundary Yang-Baxter, crossing, unitarity and bootstrap, to arrive at the amplitudes for reflection of kinks off the boundary. For the soliton/antisoliton doublet, this amplitude can can be written [1]:

$$
\mathcal{R}(\theta)=X(\theta, \xi, k)\left(\begin{array}{cc}
\cos (\xi-i \lambda \theta) & -\frac{k}{2} \sin (2 i \lambda \theta) \\
-\frac{k}{2} \sin (2 i \lambda \theta) & \cos (\xi+i \lambda \theta)
\end{array}\right)
$$


where $k$ and $\xi$ are parameters, and $\lambda \equiv \frac{8 \pi}{\beta^{2}}-1=\frac{1}{2}$ at the supersymmmetry point 3 . The parameter $\xi$ is simply related to $\Phi_{0}$ in (5.2) [1],26]:

$$
\xi=\frac{4 \pi}{\beta}\left(\Phi_{0}-\frac{2 \pi}{\beta} \operatorname{Int}\left[\frac{\beta \Phi_{0}}{2 \pi}+\frac{1}{2}\right]\right) .
$$

The parameter $k$ is a complicated function of $\Phi_{0}$ and the boundary mass $m$. The Dirichlet boundary conditions, $\left.\Phi\right|_{x=0}=\Phi_{0}$, corresponds to setting $k=0$, while Neumann boundary conditions correspond to taking $\xi=\Phi_{0}=0$, and $k=[\sin (\pi \lambda / 2)]^{-1}=\sqrt{2}$ at the supersymmetry point [1]. In the massless bulk limit, [27], the parameter $\xi$ (or $\Phi_{0}$ ) becomes a trivial (and irrelevant) shift of the free boson $\Phi$, and the reflection matrix becomes:

$$
\mathcal{R}(\theta)=\tilde{X}\left(\theta-\theta_{B}\right)\left(\begin{array}{cc}
e^{\lambda\left(\theta-\theta_{B}\right) / 2} & i e^{-\lambda\left(\theta-\theta_{B}\right) / 2} \\
i e^{-\lambda\left(\theta-\theta_{B}\right) / 2} & e^{\lambda\left(\theta-\theta_{B}\right) / 2}
\end{array}\right),
$$

where $\theta_{B}$ is a parameter related to the boundary mass scale. For Dirichlet boundary conditions, $\theta_{B} \rightarrow-\infty$, while for Neumann boundary conditions, $\theta_{B} \rightarrow+\infty$.

To have supersymmetry in the exact quantum model, the supercharges must "commute" with the reflection matrix. More precisely, one should be able to act with a supersymmetry before or after reflection, and obtain the same result. This means that we must seek a linear combination, $\mathcal{Q}(\theta)$, of the supersymmetry generators that satisfies:

$$
\mathcal{Q}(\theta) \mathcal{R}(\theta)=\mathcal{R}(\theta) \mathcal{Q}(-\theta)
$$

The minus sign on the right-hand-side comes from the fact that a kink of rapidity $\theta$ reflects as a kink of rapidity $-\theta$. For general values of $k$ and $\xi$, there is exactly one solution to this equation:

$$
\mathcal{Q}(\theta)=e^{+i \xi}\left(\mathcal{Q}_{+}+q^{-1} \widetilde{\mathcal{Q}}_{-}\right)+e^{-i \xi}\left(\mathcal{Q}_{-}+q^{-1} \widetilde{\mathcal{Q}}_{+}\right)
$$

For Dirichlet boundary conditions one finds two supercharges:

$$
\widehat{\mathcal{Q}}_{ \pm}(\theta)=\mathcal{Q}_{ \pm}+q^{-1} e^{\mp 2 i \xi} \widetilde{\mathcal{Q}}_{ \pm}
$$

and these have eigenvalues \pm 2 with respect to the (conserved) topological charge, $\mathcal{T}$. When the bulk is massless one still generically finds only one supersymmetry, and this is given by 5.15 with $\xi=0$. If one also imposes Dirichlet boundary conditions one finds two supersymmetries, given by (5.16) with $\xi=0$. For Neumann boundary conditions (with a massless bulk) one also finds two supersymmetries, and these are:

$$
\widehat{\mathcal{Q}}_{ \pm}^{\prime}(\theta)=\mathcal{Q}_{ \pm}+q^{-1} \widetilde{\mathcal{Q}}_{\mp}
$$

It should also be noted that these two supersymmetries still commute with the reflection matrix (5.11) in the $k \rightarrow \infty$ limit even when the bulk is massive.

3 Do not confuse this parameter, $\lambda$, with that of (4.1). 


\subsection{Multi-solitons states and the co-product}

While I have identified certain linear combinations of supercharges that commute appropriately with the boundary reflection matrix, there is an intrinsic danger that such linear combinations of quantum group generators may not be respected by the co-product, and so the symmetry algebra may not be well-defined on multi-particle states.

The action of the co-product on the supersymmetry generators (5.4) is:

$$
\begin{aligned}
& \Delta\left(\mathcal{Q}_{ \pm}\right)=\mathcal{Q}_{ \pm}\left(\theta_{1}\right) \otimes 1+q^{ \pm H} \otimes \mathcal{Q}_{ \pm}\left(\theta_{2}\right) \\
& \Delta\left(\widetilde{\mathcal{Q}}_{ \pm}\right)=\widetilde{\mathcal{Q}}_{ \pm}\left(\theta_{1}\right) \otimes 1+q^{\mp H} \otimes \widetilde{\mathcal{Q}}_{ \pm}\left(\theta_{2}\right)
\end{aligned}
$$

where $\theta_{j}$ is the rapidity of the $j^{\text {th }}$ kink. One sees that the linear combination (5.17) transforms consistently under the co-product, whereas the combinations (5.16) and (5.15) do not: they get different factors of $q^{ \pm H}$ for different terms in the linear combination. Thus, the terms that make up the conserved charge will be weighted by phases that depend upon the total topological charge of the state. It is perfectly reasonable for the form of a conserved fractional spin charge to depend upon the total topological charge of the state, and this will not cause a problem provided the topological charge be conserved. Certainly the bulk $S$-matrix conserves topological charge, but the reflection matrix (5.11) violates topological charge, except at the Dirichlet point. This violation is always in multiples of \pm 2 , and the relative phases in the co-product are $q^{ \pm 2 H}$ (i.e. from $q^{ \pm H}$ to $q^{\mp H}$ ), and so the violation of topological charge by boundary reflection only involves factors of $q^{ \pm 4}$. At the supersymmetry point this is not a problem, since $q^{ \pm 4}=1$.

The conclusion is that all the supercharges described in the previous section extend consistently to multi-particle states in the presence of the boundary. However, if one wishes to consider the more general Coulomb gas, with arbitrary values of $q$, then the analogue of (5.15) will not be a symmetry of the theory. Indeed, one will only have a consistent fractional spin symmetry either at at the Dirichlet point, where topological charge is conserved, or where the boundary reflection matrix is anti-diagonal, and there are two symmetries given by $(5.17)$.

\subsection{Bogolmolnyi bounds}

Since the exact quantum theory described by the structureless reflection matrix of [1] still has $N=1$ supersymmetry, one can use this to place bounds upon the energy of the ground state. 
From the positivity of the expectation value of $\left\{\mathcal{Q}^{\dagger}, \mathcal{Q}\right\}$, one finds that the following operator must have non-negative expectation value:

$$
\frac{|\lambda|}{2 \pi}\left[\left(\mathcal{P}_{+}+\mathcal{P}_{-}\right)+\left(2 \cos (2 \xi)-e^{+2 i \xi} q^{+2 \mathcal{T}}-e^{-2 i \xi} q^{-2 \mathcal{T}}\right)\right]
$$

If the topological charge, $\mathcal{T}$, is not conserved then the $\mathcal{T}$ dependent terms in the foregoing must be interpreted as part of the Hamiltonian, and one simply finds that the hamiltonian has non-negative expectation value. If, however, one has Dirichlet boundary conditions, the ground state will have topological charge given by (5.7). If $\Phi \rightarrow 0$ as $x \rightarrow-\infty$, then the topological charge of the ground state is $\mathcal{T}_{0}=\frac{\beta}{2 \pi} \Phi_{0}$. From (5.12) one sees that $\xi=\frac{8 \pi^{2}}{\beta^{2}} \mathcal{T}_{0}$ (provided $-\pi<\beta \Phi_{0}<\pi$ ), and using $q=-e^{i \pi\left(1-8 \pi / \beta^{2}\right)}=e^{-i 8 \pi^{2} / \beta^{2}}$, one obtains $q^{ \pm 2 \mathcal{T}_{0}}=e^{\mp 2 i \xi}$. Using this in (5.19) one obtains a lower bound on the energy of the ground state:

$$
E / M_{0} \geq \sin ^{2}(\xi)=\sin ^{2}\left(\frac{8 \pi^{2}}{\beta^{2}} \mathcal{T}_{0}\right)=\sin ^{2}\left(\frac{3 \pi}{2} \mathcal{T}_{0}\right)
$$

where $M_{0}$ is the mass of the soliton.

It is amusing to compare this with the classical result. If one seeks the lowest energy configuration with $\Phi \rightarrow 0$ as $x \rightarrow-\infty$, and $\left.\Phi\right|_{x=0}=\Phi_{0}$, one finds that it is a section of the single soliton solution, and if one computes the energy, one finds $E=M_{0} \sin ^{2}\left(\frac{\pi}{2} \mathcal{T}_{0}\right)$, where $\mathcal{T}_{0}=\frac{\beta \Phi_{0}}{2 \pi}$ and $M_{0}=\frac{8 M}{\beta^{2}}$ is the classical soliton mass.

The bound (5.20) is saturated if and only if the supercharge annihilates the vacuum state. For a real supersymmetry it is quite possible to get dynamical supersymmetry breaking [28], and thus this bound may not be saturated. In real supersymmetric LandauGinzburg theories, for example, one finds that one can always formally solve $\mathcal{Q} \mid 0>=0$, but the solution is not a normalizable wave-function when the superpotential is odd, or equivalently, when the index of the theory is even. On the other hand, if the model possesses a complex, or holomorphic, $N=2$ supersymmetry, then the solutions to $\mathcal{Q}_{ \pm} \mid 0>=0$ are always normalizable. The bulk sine-Gordon model has a complex $N=2$ supersymmetry, and for Dirichlet boundary conditions, this complex structure is preserved $⿴$, and the supersymmetry will not be broken. It is somewhat less clear whether the $N=1$ supersymmetry of the more general boundary conditions will be broken.

\footnotetext{
4 This will be discussed further in section 7 .
} 


\subsection{Comments}

For the boundary reflection matrix (5.11), I have shown that two supersymmetries are preserved either when one imposes Dirichlet boundary conditions, or when the bulk is massless and one imposes Neumann boundary conditions. Otherwise, the supersymmetry is reduced to $N=1$. These results are in apparent conflict with those of section 4, which indicate that one should be able to preserve $N=2$ supersymmetry for non-trivial boundary conditions. While this conflict might be partially resolved by the effects of boundary contributions of topological charges, there is still a conflict in the limit where the bulk is massless. In this limit there are no topological charges, and yet (5.11) still gives rise to only $N=1$ supersymmetry.

The key to understanding this discrepancy is a primary assumption in the derivation of the boundary reflection matrix [1], and in the imposition of (5.14). That is, to arrive at these results it was assumed that the boundary had no structure. In terms of scattering theory, this means that the boundary has no stable states, and transforms trivially under all the symmetry algebras. In terms of field theory, the concept is rather more vague, but essentially it is supposed to conjure the idea that either the boundary action contains no new degrees of freedom, or, if the boundary action does have new degrees of freedom, their evolution will be trivial in that it is completely determined by the evolution of the boundary values of bulk fields. At any rate, the amount of supersymmetry will obviously depend upon the boundary action, and, as we will see, certain very particular choices lead to $N=2$ supersymmetry, while others lead generically to $N=1$ supersymmetry.

\section{6. $N=2$ supersymmetric boundary reflection matrices}

The simplest method of introducing non-trivial boundary structure given a known

structureless exact reflection matrix, $R_{a}^{b}(\theta)$, is to formally glue a particle to the boundary国 [1,29]. To be explicit, one thinks of the new boundary as a combination of the known boundary and a particle of formal rapidity $\zeta$ running parallel to the boundary (see Fig.2). The rapidity of the boundary particle is formal since this particle is never considered as hitting the boundary - the particle is simply "glued" to the boundary forever. Let

${ }^{5}$ I am grateful to P. Fendley for explaining this process to me. 
$\alpha, \beta, \ldots$ denote the species of boundary particle, and let $a, b, c, \ldots$ denote the species of bulk particle. The new boundary reflection matrix is then given by

$$
\widetilde{R}_{a \alpha}^{b \beta}(\theta)=S_{a \alpha}^{c \gamma}(\theta-\zeta) R_{c}^{d}(\theta) S_{\alpha d}^{\beta b}(\theta+\zeta)
$$

The matrix $\widetilde{R}_{a \alpha}^{b \beta}$ can now be viewed as the reflection matrix for a boundary that has states $\alpha, \beta, \ldots$ (see Fig.2). This matrix satisfies boundary Yang-Baxter, unitarity, crossing and bootstrap as a consequence of the fact that $S_{a \alpha}^{b \beta}$ and $R_{a}^{b}$ satisfy such conditions. Note that $\widetilde{R}_{a \alpha}^{b \beta}$ has one more parameter, $\zeta$, compared to the original structureless reflection matrix. The idea now is to consider the boundary sine-Gordon model at the special points where there is $N=2$ supersymmetry. At such points there are no free parameters in $R_{a}^{b}$, but the decorated reflection matrix of (6.1) will have exactly one parameter, which can be interpreted as the boundary mass scale. Moreover, since each term on the right-handside of (6.1) commutes with the two supersymmetry generators, the resulting boundary reflection matrix will do so as well. In the process of checking the commutation of $\widetilde{R}_{a \alpha}^{b \beta}$ with the supersymmetry, one of course finds that the boundary transforms as a supersymmetry doublet of rapidity $\zeta$.

This process can obviously be generalized by gluing more particles to the boundary, and in principle there will be a parameter for each particle. In practice, once one fuses these boundary particles into higher multiplets, one might find some redundancy. For simplicity, I will only consider the gluing of a single particle here.
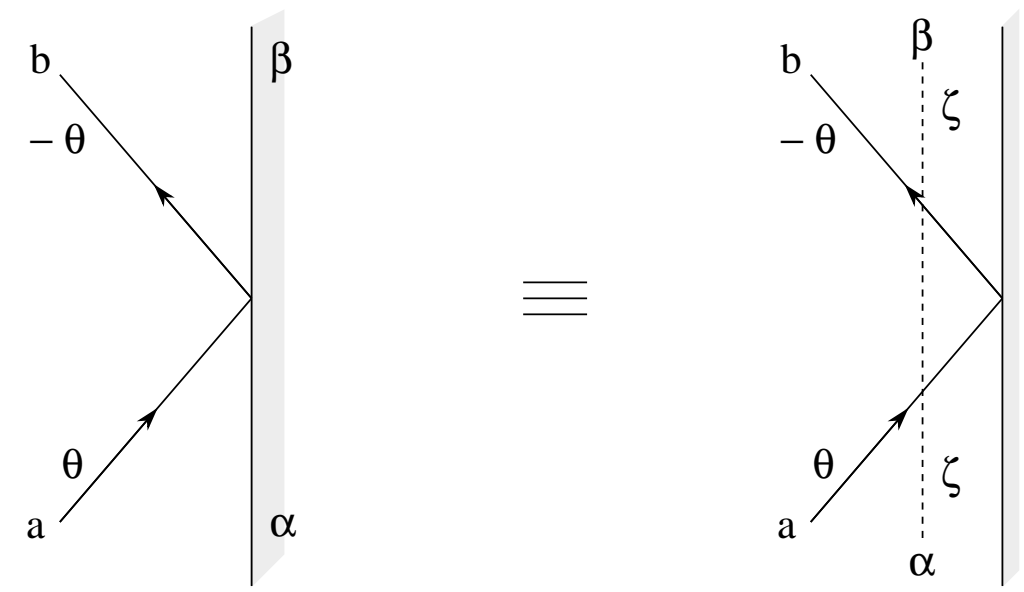

Fig.2: Obtaining boundary reflection matrices by gluing a particle of rapidity $\zeta$ to the boundary. 


\subsection{Reflection matrices preserving topological charge}

Consider first the structureless reflection matrix (5.11) with $k=0$. The bulk $S$-matrix has the form:

$$
\begin{aligned}
a(\theta) & \equiv S_{++}^{++}(\theta)=S_{--}^{--}(\theta)=Z(\theta) \sin (\lambda(\pi+i \theta)) \\
b(\theta) & \equiv S_{+-}^{+-}(\theta)=S_{-+}^{-+}=-Z(\theta) \sin (i \lambda \theta) \\
c(\theta) & \equiv S_{-+}^{+-}(\theta)=S_{+-}^{-+}=Z(\theta) \sin (\lambda \pi)
\end{aligned}
$$

where $\lambda=\frac{8 \pi}{\beta^{2}}-1$, and $Z(\theta)$ is a well known normalization factor [25]. From this one obtains a reflection matrix with the following non-vanishing terms:

$$
\begin{array}{r}
\widetilde{R}_{ \pm \pm}^{ \pm \pm}(\theta)=Y(\theta, \xi, \zeta) a(\theta+\zeta) a(\theta-\zeta) \cos (\xi \mp i \lambda \theta), \\
\widetilde{R}_{ \pm \mp}^{ \pm \mp}(\theta)=Y(\theta, \xi, \zeta)[b(\theta+\zeta) b(\theta-\zeta) \cos (\xi \mp i \lambda \theta)+ \\
c(\theta+\zeta) c(\theta-\zeta) \cos (\xi \pm i \lambda \theta)], \\
\widetilde{R}_{\mp \pm}^{ \pm}(\theta)=Y(\theta, \xi, \zeta)[b(\theta+\zeta) c(\theta-\zeta) \cos (\xi \pm i \lambda \theta)+ \\
b(\theta-\zeta) c(\theta+\zeta) \cos (\xi \mp i \lambda \theta)],
\end{array}
$$

where $Y(\theta, \xi, \zeta)=Z(\theta+\zeta) Z(\theta-\zeta) X(\theta, \xi, k=0)$. One can now explicitly verify that, provided that one has $q^{4}=1$, this reflection matrix commutes appropriately with the action of two supercharges of the form (5.16) but with the sign of the second term in (5.16) reversed. The reason for this sign reversal and the constraint $q^{4}=1$ is precisely because one needs consistency with the co-product as described in section 5.3.

\subsection{Reflection matrices from anti-diagonal scattering}

The other way to get $N=2$ supersymmetry is to use the generators (5.17), which commute with (5.11) in the $k \rightarrow \infty$ limit. The bulk does not need to be massless for this to be true, but in the massless limit this corresponds to Neumann boundary conditions. I will first consider the more general problem with a massive bulk.

The result of the fusion procedure outlined above is a reflection matrix whose non-zero entries are:

$$
\begin{aligned}
\widetilde{R}_{ \pm \pm}^{\mp \pm}(\theta) & =\hat{Y}(\theta, \zeta) a(\theta-\zeta) b(\theta+\zeta), \\
\widetilde{R}_{ \pm \pm}^{ \pm \mp}(\theta) & =\hat{Y}(\theta, \zeta) a(\theta-\zeta) c(\theta+\zeta), \\
\widetilde{R}_{ \pm \mp}^{ \pm \pm}(\theta) & =\hat{Y}(\theta, \zeta) a(\theta+\zeta) c(\theta-\zeta), \\
\widetilde{R}_{\mp \pm}^{ \pm \pm}(\theta) & =\hat{Y}(\theta, \zeta) a(\theta+\zeta) b(\theta-\zeta),
\end{aligned}
$$


where $\hat{Y}(\theta, \zeta)=\lim _{k \rightarrow \infty}\left[-\frac{k}{2} \sin (2 i \lambda \theta) X(\theta, k, \xi) Z(\theta+\zeta) Z(\theta-\zeta)\right]$.

It should be noted that are no conserved $U(1)$ charges for either this reflection matrix, or for the associated bulk scattering problem. Given the $k \rightarrow \infty$ limit of (5.11) one might have hoped that there would be a conserved "axial" $U(1)$ : the soliton number of the rightmovers minus the soliton number of the left-movers. However, this charge is violated by the off-diagonal elements, $c(\theta)$ in (6.2), in the scattering of a left-mover off a right-mover. This violation is then transmitted through to (6.4) in the fusion procedure. A $\mathbb{Z}_{4}$ subgroup of the $U(1)$ does survive, since the axial soliton number is conserved mod 4. Since kinks have fermion number $\pm \frac{1}{2}$, this means that the reflection matrix (6.4) only violates fermion number mod 2: it therefore has the physically desirable property of reflecting bosonic states as bosonic states and fermionic states as fermionic states (unlike the general form of (5.11)). This symmetry also implies that the non-vanishing reflection matrix elements (6.4) all have an odd number of + indices (or - indices).

On the other hand, it is precisely the off-diagonal terms in left-right scattering that vanish when one takes the massless limit of the bulk scattering problem. In this limit, one sends $\theta, \zeta \rightarrow \pm \infty$ (where the sign is the same as the sign of $\theta, \zeta$ respectively). One takes this limit in such a manner that $\theta-\zeta$ remains finite, and as a result the terms $R_{ \pm \pm}^{ \pm \mp}$ of the boundary reflection matrix (6.4) vanish. There is thus a conserved charge: the sum of the bulk axial $U(1)$ charge and the boundary charge.

In this massless limit one must also be a little careful in taking the infinite rapidity limit of the supersymmetry generators (5.4). If $\theta>0$ then $\mathcal{Q}_{ \pm}$will survive, and $\widetilde{\mathcal{Q}}_{ \pm}$ will go to zero; mutatis mutandis for $\theta<0$. This is simply the statement that in the conformal limit, $\widetilde{\mathcal{Q}}_{ \pm}$must act trivially on right movers, while $\mathcal{Q}_{ \pm}$will act trivially on the left movers. Another consequence of this is that the anti-commutator $\left\{\mathcal{Q}_{ \pm}, \widetilde{\mathcal{Q}}_{\mp}\right\}$ in $(5.6)$ will vanish, as it must because of the absence of bulk topological charges. As a result, the first anti-commutator in (5.10) will also vanish in this limit.

Finally, one should note that, for the reasons outlined in section 5.3, unlike the boundary reflection matrix (6.3), the matrix (6.4) will commute with the quantum group generators (5.17) for arbitrary values of $q$. Therefore, this formulation of the boundary interaction will generalize to arbitrary Coulomb gas problems. 


\section{Manifestly supersymmetric actions for systems with boundary}

The issue of what kind of boundary conditions preserve how much supersymmetry in a manifestly bulk supersymmetric theory has been a recurrent question over the years. An associated issue is the kind of boundary conditions needed to preserve a bulk Nicolai mapping (see, for example, [30]). My intent here is to go further than simple boundary conditions, and look at quantum mechanical systems on the boundary that can be coupled to the bulk theory in such a manner that supersymmetry is preserved. These models will be motivated by the Landau-Ginzburg formulations of the integrable theories described in section 4 .

The first step to constructing a manifestly supersymmetric action is to make the elementary observation that in such an action, the boundary perturbations, defined by (4.2) and (4.3), must be fermionic operators, and so there is no way that they can be added by themselves to this action. The only option is to introduce a dimensionless boundary fermion, $b$, and use fermion bilinears. There are now two choices, $b$ can be real, or complex, with either $\{b, b\}=1$ or $\{b, b\}=\left\{b^{\dagger}, b^{\dagger}\right\}=0$, and $\left\{b, b^{\dagger}\right\}=1$. Motivated by the fact the bulk massless limit of the models of interest have a conserved $U(1)$ charge, I will take $b$ to be complex. One therefore needs to consider boundary perturbations of the form:

$$
\nu b^{\dagger} \psi^{+}+\bar{\nu} \psi^{-} b
$$

Note that if $\psi^{ \pm}$transforms under a $U(1)$ charge, then one can arrange that the action preserve that $U(1)$ by making $b$ and $b^{\dagger}$ transform appropriately.

A more standard way to think of the boundary operators, $b$ and $b^{\dagger}$, is as introducing a boundary spin degree of freedom exactly as one does in the Kondo problem. Indeed, the quantization rules for $b$ and $b^{\dagger}$ imply that they generate some (possibly time dependent) representation of the gamma matrix algebra. In the sine-Gordon model, the boundary interaction (7.1) corresponds to coupling vertex operators to spin raising and lowering operators.

For the sine-Gordon model, one can also see rather explicitly that one should add these complex boundary degrees of freedom. One considers the model as one approaches the limit of free boundary conditions. As discussed in [1], any boundary degrees of freedom will not disappear in the free limit, but they will simply decouple from the bulk and will become massless boundary excitations. These will appear as poles at $\theta=i \pi / 2$ in the boundary reflection matrix. One indeed finds such poles in all channels of the reflection 
matrix, that is, in all the amplitudes: $S \rightarrow S, A \rightarrow A, S \rightarrow A$ and $A \rightarrow S$, where $S$ and $A$ denote the soliton and anti-soliton respectively 6 . Since the soliton and the anti-soliton have fermion numbers $\pm \frac{1}{2}$, the off-diagonal poles indicate that there will be boundary excitations that carry fermion number \pm 1 . The corresponding operators are $b$ and $b^{\dagger}$.

To go much further requires one to consider more specific, manifestly supersymmetric models. The problem is then a common one in conformal field theory: there are no manifestly conformal actions for the models considered in section 4. Instead, I will start with the simplest superconformal model for which we have such an action, and then move on to $N=2$ supersymmetric Landau-Ginzburg models, which provide good descriptions of the models of section 4. This will enable me to obtain the exact boundary actions and boundary superpotentials corresponding to the boundary reflection matrices of section 6.2.

\subsection{Non-trivial, supersymmetric boundary interactions}

Consider the free $N=2$ superconformal model with a complex boson and a complex Dirac fermion. This model has central charge $c=3$. The (Euclidean) action will be taken to be:

$$
\begin{aligned}
\int_{-\infty}^{0} d x \int_{-\infty}^{\infty} d y[ & \left.\left.-\left(\partial_{x} \phi\right)\left(\partial_{x} \bar{\phi}\right)-\left(\partial_{y} \phi\right)\left(\partial_{y} \bar{\phi}\right)+\frac{i}{2}\left(\bar{\lambda} \gamma^{\mu} \partial_{\mu} \lambda-\left(\partial_{\mu} \bar{\lambda}\right) \gamma^{\mu} \lambda\right)\right)\right] \\
& +\int_{-\infty}^{\infty} i\left(b^{\dagger} \frac{d}{d y} b\right)-\left.\frac{1}{2}\left(\bar{\lambda} \gamma^{*} \lambda\right)\right|_{x=0} d y
\end{aligned}
$$

where $x=x^{0}, y=x^{1}, \bar{\lambda}$ is the hermitian conjugate of $\lambda$, and

$$
\gamma^{0}=\left(\begin{array}{cc}
0 & i \\
-i & 0
\end{array}\right) \quad \gamma^{1}=\left(\begin{array}{cc}
1 & 0 \\
0 & -1
\end{array}\right) \quad \gamma *=-i \gamma^{0} \gamma^{1}=\left(\begin{array}{ll}
0 & 1 \\
1 & 0
\end{array}\right)
$$

A boundary term has already been added to the standard free action in (7.2). This is motivated by the boundary action for the Ising model (see, for example, [1]), and it incorporates the free boundary fermions. The boundary term involving $\lambda$ has been included to simplify computations, and in particular, it leads naturally to the free boundary conditions for the bulk fermion. One should also note that without this $\bar{\lambda} \gamma^{*} \lambda$ term, the action is invariant under both the left and right handed fermion number symmetries independently, whereas

6 The poles in the off-diagonal part of the $S$-matrix were not so much of a concern in [1] since the focus was mainly on values of the coupling for which these poles are cancelled by zeroes in the numerator. At the supersymmetry point there are no such cancellations. 
one knows that only one linear combination of these $U(1)$ symmetries will survive on the half-space. The boundary term has effectively selected which $U(1)$ symmetry survives, and this is the axial $U(1)$ :

$$
\lambda_{j} \rightarrow e^{i \nu} \lambda_{j}, \quad \bar{\lambda}_{j} \rightarrow e^{-i \nu} \bar{\lambda}_{j}
$$

where $\lambda_{j}$ are the components of $\lambda$.

The variation of $(7.2)$ yields the bulk equations of motion, along with the boundary terms:

$$
\begin{array}{r}
-\left.\int_{-\infty}^{\infty}\left[\left(\partial_{x} \bar{\phi}\right)(\delta \phi)+\left(\partial_{x} \phi\right)(\delta \bar{\phi})\right]\right|_{x=0}-i\left[\left(\frac{d}{d y} b^{\dagger}\right) \delta b+\left(\frac{d}{d y} b\right) \delta b^{\dagger}\right] \\
+\left.\frac{1}{2}\left[\left(\bar{\lambda}_{1}-\bar{\lambda}_{2}\right)\left(\delta \lambda_{1}+\delta \lambda_{2}\right)-\left(\lambda_{1}-\lambda_{2}\right)\left(\delta \bar{\lambda}_{1}+\delta \bar{\lambda}_{2}\right)\right]\right|_{x=0} d y
\end{array}
$$

Requiring that (7.4) vanish imposes Neumann, Dirichlet, or a combination of Neumann and Dirichlet conditions on the real and imaginary parts of the boson, along with analogous boundary conditions on the fermion.

The bulk part of the action (7.2) also has an $N=2$ superconformal symmetry, which can be written in terms of components as:

$$
\begin{array}{rlrl}
\delta \phi & =-\left(\lambda_{1} \bar{\alpha}_{1}+\lambda_{2} \bar{\alpha}_{2}\right), & \delta \bar{\phi}=\left(\bar{\lambda}_{1} \alpha_{1}+\bar{\lambda}_{2} \alpha_{2}\right), \\
\delta \lambda_{1} & =-\left[\partial_{x} \phi-i \partial_{y} \phi\right] \alpha_{2}, & \delta \bar{\lambda}_{1} & =-\left[\partial_{x} \bar{\phi}+i \partial_{y} \bar{\phi}\right] \bar{\alpha}_{2}, \\
\delta \lambda_{2} & =\left[\partial_{x} \phi+i \partial_{y} \phi\right] \alpha_{1}, & \delta \bar{\lambda}_{2} & =\left[\partial_{x} \bar{\phi}-i \partial_{y} \bar{\phi}\right] \bar{\alpha}_{1} ;
\end{array}
$$

where $\alpha_{1}, \alpha_{2}, \bar{\alpha}_{1}$ and $\bar{\alpha}_{2}$ are the complex fermionic supersymmetry parameters. If one uses this in the action (7.2), and assumes that $\delta b=\delta b^{\dagger}=0$, then one generates the following boundary terms:

$$
\begin{aligned}
-\frac{1}{2} \int_{-\infty}^{\infty}\left[\left(\partial_{x} \phi\right)\left(\bar{\lambda}_{1}-\bar{\lambda}_{2}\right)-i\left(\partial_{y} \phi\right)\left(\bar{\lambda}_{1}+\bar{\lambda}_{2}\right)\right]\left(\alpha_{1}-\alpha_{2}\right) \\
-\left[\left(\partial_{x} \bar{\phi}\right)\left(\lambda_{1}-\lambda_{2}\right)-i\left(\partial_{y} \bar{\phi}\right)\left(\lambda_{1}+\lambda_{2}\right)\right]\left(\bar{\alpha}_{1}-\bar{\alpha}_{2}\right) .
\end{aligned}
$$

To preserve the supersymmetry in the system with boundary, one must choose boundary conditions that cause (7.4) and (7.6) to vanish 7 . Further restrictions might follow from requiring that the boundary conditions are consistent with (7.5). Obvious solutions are:

(i) Dirichlet boundary conditions for the bosons, $\left.\phi\right|_{x=0}=$ const, along with $\alpha_{1}=\alpha_{2}=\alpha$. Requiring consistency with $(7.5)$ means that we must also impose $\left.\left(\lambda_{1}+\lambda_{2}\right)\right|_{x=0}=0$.

7 It is in making these choices that one ordinarily selects which $U(1)$ fermion number symmetry will be preserved in the boundary theory. 
(ii) Neumann boundary conditions for the bosons, $\left.\partial_{x} \phi\right|_{x=0}=0$, along with $\alpha_{1}=\alpha_{2}=\alpha$. Requiring consistency with (7.5) means that we must also impose $\left.\left(\lambda_{1}-\lambda_{2}\right)\right|_{x=0}=0$.

(iii) A combination of Dirichlet and Neumann conditions, in which one takes $\partial_{x}(\phi+$ $\bar{\phi})\left.\right|_{x=0}=0$, and $\left.(\phi-\bar{\phi})\right|_{x=0}=$ const. One then finds that one must impose $\left.\left(\lambda_{j}+\bar{\lambda}_{j}\right)\right|_{x=0}=0$, and $\alpha_{j}=\bar{\alpha}_{j}, j=1,2$.

The first two choices preserve a complex $N=2$ superconformal algebra, with parameters $\alpha$ and $\bar{\alpha}$, while the last alternative is apparently a real $N=2$ algebra, but one can find a new basis in which it will be a complex supersymmetry.

The boundary perturbation analogous to (7.1) is given by the same expression, but with $\psi^{ \pm}$replaced by $\left(\lambda_{1}+\lambda_{2}\right)\left(\frac{\partial^{2} V}{\partial \phi^{2}}\right)$ and $\left(\bar{\lambda}_{1}+\bar{\lambda}_{2}\right)\left(\frac{\partial^{2} \bar{V}}{\partial \phi^{2}}\right)$ respectively, where $V$ is some superpotential function. Supersymmetry then requires a boundary action of the form:

$$
\frac{1}{2} \int_{-\infty}^{\infty}\left(\frac{\partial^{2} V}{\partial \phi^{2}}\right) b^{\dagger}\left(\lambda_{1}+\lambda_{2}\right)-\left(\frac{\partial^{2} \bar{V}}{\partial \bar{\phi}^{2}}\right) b\left(\bar{\lambda}_{1}+\bar{\lambda}_{2}\right)+\left|\frac{\partial V}{\partial \phi}\right|^{2} d y
$$

Combining the variation of this with $(7.4)$, one finds that the equations of motion of the boundary degrees of freedom are:

$$
\begin{aligned}
\left.\left(\lambda_{1}-\lambda_{2}\right)\right|_{x=0} & =\left.b\left(\frac{\partial^{2} \bar{V}}{\partial \bar{\phi}^{2}}\right)\right|_{x=0} ; \quad \frac{d}{d y} b=-\left.\frac{i}{2}\left(\lambda_{1}+\lambda_{2}\right)\left(\frac{\partial^{2} V}{\partial \phi^{2}}\right)\right|_{x=0} ; \\
\left.\left(\partial_{x} \phi\right)\right|_{x=0} & =\left.\frac{1}{2}\left(\frac{\partial V}{\partial \phi}\right)\left(\frac{\partial^{2} \bar{V}}{\partial \bar{\phi}^{2}}\right)\right|_{x=0}-\left.b\left(\bar{\lambda}_{1}+\bar{\lambda}_{2}\right)\left(\frac{\partial^{3} \bar{V}}{\partial \bar{\phi}^{3}}\right)\right|_{x=0} ;
\end{aligned}
$$

along with the complex conjugates of these equations.

This model is also invariant under a complex $N=2$ supersymmetry. All one needs to do is set $\alpha_{1}=\alpha_{2}=\alpha$ in (7.5), and use (7.5) along with:

$$
\delta b=-\left.\left(\frac{\partial V}{\partial \phi}\right)\right|_{x=0} \alpha ; \quad \delta b^{\dagger}=-\left.\left(\frac{\partial \bar{V}}{\partial \bar{\phi}}\right)\right|_{x=0} \bar{\alpha}
$$

This $N=2$ supersymmetry is consistent with $U(1)$ symmetry (7.3) provided that the boundary fermions and supersymmetry parameters transform according to:

$$
\alpha \rightarrow e^{i \nu} \alpha, \quad \bar{\alpha} \rightarrow e^{-i \nu} \bar{\alpha}, \quad b \rightarrow e^{i \nu} b, \quad b^{\dagger} \rightarrow e^{-i \nu} b^{\dagger}
$$

\subsection{Introducing a bulk superpotential}

Consider now what happens when the bulk becomes a massive Landau-Ginzburg theory. That is, temporarily ignore all boundary terms, and add the following to the bulk Euclidean action:

$$
\int_{-\infty}^{0} d x \int_{-\infty}^{\infty} d y\left[\left(\frac{\partial^{2} W}{\partial \phi^{2}}\right) \lambda_{1} \lambda_{2}-\left(\frac{\partial^{2} \bar{W}}{\partial \bar{\phi}^{2}}\right) \bar{\lambda}_{1} \bar{\lambda}_{2}-\left|\frac{\partial W}{\partial \phi}\right|^{2}\right]
$$


for some scalar superpotential, $W$. To preserve the bulk supersymmetry one must add the following terms to the supersymmetry transformations of the fermions:

$$
\delta_{W} \lambda_{i}=\epsilon^{i j}\left(\frac{\partial \bar{W}}{\partial \bar{\phi}}\right) \bar{\alpha}_{j}, \quad \delta_{W} \bar{\lambda}_{i}=\epsilon^{i j}\left(\frac{\partial W}{\partial \phi}\right) \alpha_{j}
$$

where $\epsilon^{i j}=-\epsilon^{i j}$ and $\epsilon^{12}=+1$. This bulk action preserves the vector $U(1)$ symmetry:

$$
\lambda_{1} \rightarrow e^{i \nu} \lambda_{1}, \quad \lambda_{2} \rightarrow e^{-i \nu} \lambda_{2} ; \quad \bar{\lambda}_{1} \rightarrow e^{i \nu} \bar{\lambda}_{1}, \quad \bar{\lambda}_{2} \rightarrow e^{-i \nu} \bar{\lambda}_{2}
$$

with the supersymmetry parameters transforming in a similar manner (but with the subscripts 1,2 interchanged).

If $W$ is quasi-homogeneous, that is, if

$$
W\left(a^{\omega} \phi\right)=a W(\phi)
$$

for some $\omega$ and any value of $a$, then this action also preserves an $R$-symmetry. Namely, it is invariant under:

$$
\begin{aligned}
\phi & \rightarrow e^{i \omega \nu} \phi, \quad \bar{\phi} \rightarrow e^{-i \omega \nu} \bar{\phi} \\
\lambda_{j} & \rightarrow e^{-\frac{i}{2}(1-2 \omega) \nu} \lambda_{j}, \quad \bar{\lambda}_{j} \rightarrow e^{+\frac{i}{2}(1-2 \omega) \nu} \bar{\lambda}_{j}, \quad j=1,2 \\
\alpha & \rightarrow e^{-\frac{i}{2} \nu} \alpha, \quad \bar{\alpha} \rightarrow e^{+\frac{i}{2} \nu} \bar{\alpha} .
\end{aligned}
$$

Now consider the boundary theory. There are two different ways of getting $N=2$ supersymmetry, and these correspond to the two distinct $N=2$ algebras described earlier. One can take:

$$
\text { (i) } \quad \alpha_{j}=\bar{\alpha}_{j}, j=1,2 \quad \text { or } \quad \text { (ii) } \quad \alpha=\alpha_{1}=\alpha_{2}, \quad \bar{\alpha}=\bar{\alpha}_{1}=\bar{\alpha}_{2} .
$$

The second choice is the more interesting topologically since the supersymmetry transformations involving $W$ and those involving derivatives of fields remain independent. Amongst other things, this is crucial to relating the $N=2$ supersymmetric theory to a topological Landau-Ginzburg theory [31]. The first choice is more akin to a real supersymmetry. One can also verify directly from the supercurrents [10] that the first superalgebra has the form (5.9), whereas the second choice leads to a bulk superalgebra of the form (5.10). Indeed, the topological charge for the Landau-Ginzburg model is [8,9]:

$$
\mathcal{T}=\int \partial_{x} W d x,
$$


and, for example, one can write the supercurrent that generates the transformation with parameter $\alpha$ as:

$$
\mathcal{G}_{+}=\bar{\lambda}_{1}\left(\partial_{x} \phi+i \partial_{y} \phi\right)-\bar{\lambda}_{2}\left(\partial_{x} \phi-i \partial_{y} \phi\right)+\left(\lambda_{2}-\lambda_{1}\right)\left(\frac{\partial W}{\partial \bar{\phi}}\right)
$$

Here I am taking the convention that the short-distance expansion of the fermions is normalized to $\bar{\lambda}_{1}(z) \lambda_{2}(w) \sim \frac{1}{z-w}$ and $\bar{\lambda}_{2}(\bar{z}) \lambda_{1}(\bar{w}) \sim \frac{1}{\bar{z}-\bar{w}}$. Taking the operator product of $\mathcal{G}_{+}$with itself generates simple pole terms whose net contribution to the anti-commutator of this supercharge with itself is $\int_{-\infty}^{0} 4\left(\frac{\partial W}{\partial \phi}\right)\left(\partial_{x} \phi\right) d x=\left.4 W\right|_{x=0}$.

To make the boundary theory consistent, one must once again ensure that all the boundary variations vanish, and then check consistency with the combination of (7.5) and (7.12). The ordinary variation of (7.11) does not give any new boundary terms in (7.4), but the supersymmetry variation of $(7.11)$ does give rise to the following additional boundary term:

$$
\left.\frac{1}{2} \int_{-\infty}^{\infty}\left[\left(\frac{\partial W}{\partial \phi}\right)\left(\lambda_{1}+\lambda_{2}\right)\left(\alpha_{1}+\alpha_{2}\right)-\left(\frac{\partial \bar{W}}{\partial \bar{\phi}}\right)\left(\bar{\lambda}_{1}+\bar{\lambda}_{2}\right)\left(\bar{\alpha}_{1}+\bar{\alpha}_{2}\right)\right]\right|_{x=0} d y
$$

Suppose for the moment that boundary potential $V$ to zero. If one looks for supersymmetry of type (ii), then to remove the terms in (7.19) it suffices to set either $\left.\left(\lambda_{1}+\lambda_{2}\right)\right|_{x=0}=0$ or $\left.\frac{\partial W}{\partial \phi}\right|_{x=0}=0$. However, requiring that (7.4) vanish and checking consistency with (7.5) and (7.12) leads to the Dirichlet conditions: $\phi_{x=0}=\phi_{0}$ and $\left.\left(\lambda_{1}+\lambda_{2}\right)\right|_{x=0}=0$. Note that these boundary conditions break the vector-like $U(1)$ symmetry, but if $W$ is quasi-homogeneous, then they preserve the $R$-symmetry.

If one looks for a purely Neumann boundary conditions, then the supersymmetry transformations lead one to impose:

$$
\begin{aligned}
\alpha_{1} & =\alpha_{2}=\bar{\alpha}_{1}=\bar{\alpha}_{2} ;\left.\quad\left(\lambda_{1}-\lambda_{2}\right)\right|_{x=0}=0 ; \\
\left.\partial_{x} \phi\right|_{x=0} & =\left.\left(\frac{\partial \bar{W}}{\partial \bar{\phi}}\right)\right|_{x=0} ;\left.\quad \partial_{x} \bar{\phi}\right|_{x=0}=\left.\left(\frac{\partial W}{\partial \phi}\right)\right|_{x=0}
\end{aligned}
$$

Note that the first of these equations means that the supersymmetry has been reduced to $N=1$. The constraints $(7.20)$ do not guarantee the vanishing of $(7.19)$. To accomplish this one needs to add another obvious boundary term:

$$
\left.\int_{-\infty}^{\infty}(W(\phi)+\bar{W}(\bar{\phi}))\right|_{x=0} d y
$$


This extra boundary term is also crucial for the bosonic boundary conditions in (7.20) to follow from the variation of the action. This term is also nothing other than the boundary contribution to the bulk topological charge (7.17). It is also amusing to note that for Dirichlet boundary conditions, where the topological charge is conserved, one has the option of adding (7.21) since it will make no difference to the boundary variation. Thus, when the topological charge is conserved, one can chose whether, or not, to include such terms in the action, and hence hamiltonian. When the topological charge is not conserved, one no longer has the option, and one must include such the topological charge terms in the action. At the same time, one also sees a reduction from complex $N=2$ to real $N=1$ supersymmetry. These boundary conditions also break all $U(1)$ symmetries, reducing any $R$-symmetry to a discrete subgroup.

If one now seeks $N=2$ supersymmetry of type (i), one is led to a combination of Neumann and Dirichlet conditions. One uses an action that includes (7.21), and one takes:

$$
\begin{array}{rlrl}
\alpha_{j} & =\bar{\alpha}_{j} ; \quad & \left.\left(\lambda_{j}+\bar{\lambda}_{j}\right)\right|_{x=0}=0 ; \quad j=1,2 ; \\
\left.(\phi-\bar{\phi})\right|_{x=0}=2 i \phi_{0} ; & {\left.\left[\partial_{x}(\phi+\bar{\phi})\right]\right|_{x=0}=\left.\left(\frac{\partial W}{\partial \phi}+\frac{\partial \bar{W}}{\partial \bar{\phi}}\right)\right|_{x=0} .}
\end{array}
$$

Note that the non-trivial boundary dynamics only involve the real part of $\phi$, and so the boundary "quantum mechanics" is that of a single real scalar field. These boundary conditions break any $R$-symmetries, but preserve the vector-like $U(1)$ symmetry $(7.13)$. (The boundary term in (7.2) that breaks this $U(1)$ vanishes identically as a consequence of the boundary conditions.)

One can now combine the foregoing with a non-trivial boundary interaction, that is, one considers an action that is the sum of (7.2), (7.7), (7.11) and (7.21). This action only possesses an $N=1$ supersymmetry. However, exactly as in section 6 , one can adjust the non-trivial boundary dynamics so as to get a type (ii) $N=2$ supersymmetry. One takes an action that is the sum of (7.2), (7.7) and (7.11), but does not include (7.21). One then cancels the variation (7.19) by the simple expedient of setting:

$$
\left.\left(\frac{\partial V}{\partial \phi}\right)\right|_{x=0}=\left.\mu W\right|_{x=0}
$$

and changing (7.9) to:

$$
\delta b=2 \bar{\mu}^{-1} \bar{\alpha}-\left.\mu \alpha W\right|_{x=0} ; \quad \delta b^{\dagger}=2 \mu^{-1} \alpha-\left.\bar{\mu} \bar{\alpha} \bar{W}\right|_{x=0}
$$


The parameter $\mu$ is an arbitrary complex number whose modulus can be thought of as the ratio of the boundary and bulk mass scales. The real and imaginary parts of $\mu$, along with the bulk mass scale are precisely the three independent parameters identified in the conformal perturbation theory of section 4. For the sake of completeness, the classical equations of motion of this $N=2$ supersymmetric model are:

$$
\begin{aligned}
\left.\left(\lambda_{1}-\lambda_{2}\right)\right|_{x=0} & =\left.2 \bar{\mu} b\left(\frac{\partial \bar{W}}{\partial \bar{\phi}}\right)\right|_{x=0}, \quad \frac{d}{d y} b=\left.\frac{i}{2} \mu\left(\lambda_{1}+\lambda_{2}\right)\left(\frac{\partial W}{\partial \phi}\right)\right|_{x=0}, \\
\left.\left(\bar{\lambda}_{1}-\bar{\lambda}_{2}\right)\right|_{x=0} & =\left.2 \mu b^{\dagger}\left(\frac{\partial W}{\partial \phi}\right)\right|_{x=0}, \quad \frac{d}{d y} b^{\dagger}=\left.\frac{i}{2} \bar{\mu}\left(\bar{\lambda}_{1}+\bar{\lambda}_{2}\right)\left(\frac{\partial \bar{W}}{\partial \bar{\phi}}\right)\right|_{x=0}, \\
\left.\partial_{x} \phi\right|_{x=0} & =-\left.\bar{\mu} b\left(\bar{\lambda}_{1}+\bar{\lambda}_{2}\right)\left(\frac{\partial^{2} \bar{W}}{\partial \bar{\phi}^{2}}\right)\right|_{x=0}+\left.2|\mu|^{2} W\left(\frac{\partial \bar{W}}{\partial \bar{\phi}}\right)\right|_{x=0} .
\end{aligned}
$$

One can easily check that these are consistent with the type (ii) $N=2$ supersymmetry transformations.

Having found a combined boundary and bulk interaction that preserves $N=2$ supersymmetry it is useful to observe that one can now add more species of boundary fermion, with whatever potentials one desires and still keep $N=2$ supersymmetry. Specifically, one can add any number of boundary fermions with couplings of the form (7.7), and with supersymmetry transformations given by (7.9). The choice of bosonic potentials for these extra fermion species is also arbitrary. Presumably only a small subset of these models will be quantum integrable.

\subsection{Comments}

There are several important things to note about this last result. First, in order to get $N=2$ supersymmetry, the boundary and bulk superpotential must be related by (7.23). This should not be a surprise given the discussion in sections 3 and 4, where the boundary integrable perturbation is obtained directly from the bulk integrable perturbation. The derivative in (7.23) may be a little surprising, but is a natural consequence of the fact that the boundary perturbation, defined by (4.2) and (7.1), only involves the action of one supercharge whereas the bulk perturbation, (4.1), involves the action of two supercharges. It is also amusing to note that for the bulk Chebyshev perturbation, the superpotential, $W$, is a polynomial of degree $k+2$. This means that the bulk bosonic potential has $k+1$ zero-energy minima, while the boundary bosonic potential has $k+2$ zero-energy minima. The constraint between boundary and bulk potential is also familiar in sine-Gordon and 
Toda theories. One should note, however, that here the bulk and boundary mass scales are independent

The next thing to observe is that the boundary topological charge terms (7.21) have been done away with completely. What has happened is that the new transformation (7.24) has introduced the following boundary terms into the supercurrents:

$$
\mathcal{G}_{b,+}=2 \mu^{-1} b-\left.\mu b^{\dagger} W\right|_{x=0} ; \quad \mathcal{G}_{b,-}=2 \bar{\mu}^{-1} b^{\dagger}-\left.b \bar{\mu} \bar{W}\right|_{x=0} .
$$

If one computes the anti-commutator each of these charges with itself, then one generates either $-\left.4 W\right|_{x=0}$ or $-\left.4 \bar{W}\right|_{x=0}$, which are precisely what one needs in order to cancel the boundary contribution of the topological terms coming from the anti-commutators of the bulk supercharges. (See the discussion following (7.17).) Thus one can reverse the foregoing perspective entirely, and take the view that the problem of boundary contributions of bulk topological charge terms has been solved by introducing $W$-dependent terms into the supersymmetry variation of the boundary fermions, and this, in turn requires the boundary dynamics described by (7.7) and (7.23).

One should also observe that because one has managed to do away with the terms (7.21), the action will now preserve the $R$-symmetry when superpotential is quasihomogeneous. Otherwise this $U(1)$ is broken generically to a $\mathbb{Z}_{2}$, (the fermion number mod 2), or perhaps a larger discrete group if the superpotential has additional symmetry.

The $N=2$ supersymmetric boundary Landau-Ginzburg theories constructed here clearly provide the effective field theories of the perturbed models described in section 4 . This correspondence is well established for the bulk, but here I have been able to get the exact effective boundary potential from the knowledge of the bulk theory and the existence of $N=2$ supersymmetry. The fact that one can preserve the $R$-symmetry of the model when the superpotential is quasi-homogeneous not only leads to the scale invariance of the interaction, and to an all important conserved $U(1)$ current, but it also means that one should be able to formulate a Coulomb gas description of this boundary model based upon the methods of [32,33, 12 .

The structure of this effective theory is also completely parallel to the scattering theory described in section 6.2. If the model is massless in the bulk then there is always $N=2$ supersymmetry, along with a conserved axial $U(1)$ symmetry. If the bulk is massive, then the axial symmetry is broken down to conservation of the fermion number mod 2 , and the supersymmetry is reduced to $N=1$. However, if one chooses exactly the right 
non-trivial boundary dynamics then one can restore the $N=2$ supersymmetry (but not the axial $U(1))$. Moreover, the process of getting ever more complicated boundary reflection matrices via the gluing procedure must coincide with the introduction of more and more boundary fermion species in the Landau-Ginzburg formulation. Indeed, introducing additional species of boundary fermion also extends the boundary Hilbert space by a spin doublet for each such fermion. One can then extract higher spin boundary states by projecting onto irreducible factors in the tensor product of the doublets. This is very reminiscent of the the Kondo problem, which can described by a resonant level model [34] in which the spin impurity is created using boundary fermions. The Kondo problem can also be described in terms of exact $S$-matrices on a system with a boundary, with the spin impurity being described in terms a boundary potential when the impurity is underscreened, or in terms of kinks when the impurity is overscreened [35].

Finally, there is something of a conundrum with the type (i) $N=2$ supersymmetry of (7.22). The indications from sections 5 and 6 are that there should be an exactly solvable model with $N=2$ supersymmetry, and a conserved vector-like charge. It seems very plausible that the effective action for this model is the one with a structureless boundary that leads to (7.22). However, it does not appear to be possible to implement this form of $N=2$ supersymmetry in the presence of non-trivial boundary interactions. Thus the effective actions corresponding to the boundary reflection matrices described in section 6.1 are, as yet, unknown.

\section{Conclusions}

In this paper it has been shown, using both field theory and exact $S$-matrix theory, that there are quantum integrable, boundary field theories that have non-trivial boundary dynamics and possess both $N=1$ and $N=2$ supersymmetry. This was done initially by using conformal perturbation theory, and these results were further substantiated by the construction of the manifestly supersymmetric Landau-Ginzburg effective actions that had all the properties that one would expect based upon conformal perturbation theory and the known results of bulk Landau-Ginzburg theory. It was also shown that one could easily construct $N=1$ supersymmetric actions with independent boundary and bulk actions, but one only gets $N=2$ supersymmetry when the boundary action is related to the bulk action in exactly the manner suggested by the conformal perturbation theory. 
A directly parallel story occurs with the construction of the exact boundary reflection matrices. The structureless reflection matrices for the sine-Gordon model only have $N=1$ supersymmetry, except at special points in the parameter space, where the supersymmetry is enhanced to $N=2$. This coincides with the results coming from simplest boundary interactions in the effective Landau-Ginzburg theory. In order to get $N=2$ supersymmetric exact reflection matrices, one can go to these special $N=2$ supersymmetric points for the known structureless reflection matrix, and then construct more complicated such matrices by decorating the boundary by gluing kinks to it. One then finds natural candidate reflection matrices for the integrable models described by the field theory. An issue that is not fully resolved here is precisely how many glued kinks correspond to the basic $N=2$ supersymmetric Landau-Ginzburg model with only one species of boundary fermion. One way to resolve this is to apply the ideas of [35], and perform a Bethe Ansatz computation to determine the boundary entropy, and then use this to determine the number of wells in the boundary potential. This is presently under investigation.

From the results of section 7.1, one sees that one can couple a free $N=2$ supersymmetric theory to an arbitrary boundary superpotential, and still preserve $N=2$ supersymmetry. However, based upon the results of section 7.2, I do not believe that this will remain true in any $N=2$ superconformal field theory. To get a specific nontrivial $N=2$ superconformal model from an effective action, one needs to use a specific quasi-homogeneous superpotential. This means that the boundary potential is fixed by the $N=2$ supersymmetry. Similarly for perturbed $N=2$ supersymmetric boundary field theories: the effective boundary potential for the quantum integrable perturbation is uniquely determined by the choice of the superconformal model, and the corresponding bulk integrable perturbation, even when the bulk remains massless. These potentials are thus known explicitly by virtue of the supersymmetry and the known form of the integrable bulk superpotentials. If one wants to have more exotic boundary interactions one must introduce more fermionic boundary degrees of freedom.

The results presented in this paper are also highly encouraging for the use Coulomb gas methods for more general boundary integrable models. In sections 5 and 6 , I identified two natural superalgebras on the half-space, and observed that one of them only had a well defined action at the supersymmetry point, whereas the other would obviously generalize to other values of the quantum group parameter, $q$. It also turned out that the latter "good" superalgebra was the one that coincided with the superalgebras that came from conformal perturbation theory and effective Landau-Ginzburg actions. As a 
result, I expect that the more general conformal perturbation theory and Coulomb gas ideas should be generalizable to boundary theories. I also anticipate that the results will be qualitatively similar to those of section 7 , in that one will have to use appropriate nontrivial boundary degrees of freedom, and that the boundary theory will probably have some qualitative multi-well effective potential that depends upon the value of $q$, the quantum group parameter 8 .

The fact that one can very probably generalize Coulomb gas methods to the halfspace, suggests that, if one introduces the proper boundary degrees of freedom, one should be able to get the correspondence between conformal field theories and Toda models to work in systems with boundary as well. Thus any discrepancy between the two approaches will occur either because one has not used exactly the correct boundary interaction, or that there is no good conformal limit to the boundary Toda potential (i.e. the vertex operators in the boundary potential do not correspond to operators in the conformal boundary spectrum).

Finally, two thoughts from extreme ends of the subject. First, it is intriguing that the boundary action of the $N=2$ supersymmetric model depends explicitly upon the bulk superpotential, and not just on some derivatives of it. That is, the bosonic boundary potential is $|W|^{2}$. In the discussion of the applications of singularity theory to $N=2$ supersymmetric models, a minor frustration was that it was hard to see a direct action of the monodromy group of the singularity on the physical states of the theory. This is basically because the physical states cared primarily about the critical points of $W$, and not about the values of $W$, whereas the most beautiful theorems of singularity theory relate to the latter. Since the infra-red limit of the boundary theory is clearly concerned with the values of $W$, it is conceivable that it might provide a clean physical realization of the action of the monodromy group of the singularity.

The other thought is that it will be very interesting to see whether the results presented here can be used in the study of monopoles or cosmic strings in $(3+1)$ dimensions. As mentioned in the introduction, treating a supersymmetric monopole as an impurity problem will result in a potentially supersymmetric $(1+1)$-dimensional boundary theory. Apart from the issue of quantum integrability, it would also be very interesting to understand, in the $(3+1)$ dimensional context, the interplay between boundary degrees of

8 H. Saleur has also come to similar conclusions by other methods, and I am grateful to him for conversations on this issue. 
freedom (excitations of the monopole), the amount of supersymmetry, and the consequent Bogolmolnyi bounds.

\section{Acknowledgements:}

I would like to thank P. Dorey, J. McCarthy, J.-B. Zuber and particularly P. Fendley and H. Saleur for valuable discussions on this work. I am also grateful to the the University of Adelaide, the Service de Physique Théorique at Saclay and to the High Energy Physics Laboratory of the University of Paris VI in Jussieu for hospitality while substantial parts of this work were done.

\section{Dedication:}

As this work was approaching completion I learned that Claude Itzykson had died. Apart from being a remarkable physicist, he was also a good friend and great colleague. I shall miss his conversation and lectures, and regret that the exclamation "excellent!" will no longer be used as an exhortation to rapidly terminate a boring discussion. I would like to dedicate this work, such as it is, to Claude's memory. 


\section{References}

[1] S. Ghoshal and A.B. Zamolodchikov, Int. J. Mod. Phys. A9 (1994) 3841, hepth/9306002.

[2] N. Andrei, K Furuya and J. Lowenstein, Rev. Mod. Phys. 55 (1983) 331;

A.M. Tsvelik and P.B. Wiegmann, Adv. Phys. 32 (1983) 453.

[3] E. Corrigan, P.E. Dorey, R.H. Rietdijk and R. Sasaki, "Affine Toda Field Theory on a Half-Line," Phys. Lett. 333B (1994) 83; DTP-94-57, hep-th/9501098;

P. Bowcock, E. Corrigan, P.E. Dorey and R.H. Rietdijk, "Classically Integrable Boundary Conditions for Affine Toda Field Theories," DTP-94-57, hep-th/9501098.

[4] S. Penati and D. Zanon "Quantum Integrability in Two-Dimensional Systems with Boundary," IFUM-490-FT, hep-th/9501105.

[5] V.A. Rubakov, Nucl. Phys. B203 (1982) 311;

C.G. Callan, Phys. Rev. D25 (1982) 2141; Phys. Rev. D26 (1982) 2058; Nucl. Phys. B212 (1983) 391;

C.G. Callan and S.R. Das, Phys. Rev. Lett. 51 (1983) 1155 .

[6] X.G. Wen, Phys. Rev. B41 (1990) 12838; Phys. Rev. B43 (1991) 11025; Phys. Rev. B44 (1991) 5708.

[7] P. Fendley, A. Ludwig and H. Saleur, Phys. Rev. Lett. 74 (1995) 3005; condmat/9408068.

[8] E. Witten and D. Olive, Phys. Lett. 78 (1978) 97.

[9] P. Fendley, S. Mathur, C. Vafa and N.P. Warner, Phys. Lett. 243B (1990) 257.

[10] W. Lerche and N.P. Warner, Nucl. Phys. B358 (1991) 571.

[11] E. Witten, Int. J. Mod. Phys. A9 (1994) 4783;

P. di Francesco and S. Yankielowicz, Nucl. Phys. B409 (1993) 18;

P. di Francesco, O. Aharony and S. Yankielowicz, Nucl. Phys. B411 (1994) 584;

T. Kawai, Y. Yamada and S.-K. Yang, Nucl. Phys. B414 (1994) 191;

T. Kawai, Phys. Lett. 342B (1995) 87; T. Kawai and S.-K. Yang, "Duality of Orbifoldized Elliptic Genera," preprint KEK-TH-409, hep-th/9408121;

M. Henningson, Nucl. Phys. B413 (1994) 73;

P. Berglund and M. Henningson, Nucl. Phys. B433 (1995) 311; "On the Elliptic Genus and Mirror Symmetry," IASSNS-HEP-94-11, hep-th/9406045.

[12] D. Nemeschansky and N.P. Warner, Phys. Lett. 329B (1994) 53; "The Refined Elliptic Genus and Coulomb Gas Formulations of $N=2$ Superconformal Coset Models," USC94-018, hep-th/9412187.

[13] S. Cecotti, P. Fendley, K. Intriligator and C. Vafa, Nucl. Phys. B386 (1992) 405;

P. Fendley and H. Saleur, Nucl. Phys. B388 (1992) 609.

[14] J.L. Cardy, Nucl. Phys. B240 (1984) 514; Nucl. Phys. B275 (1986) 200; Nucl. Phys. B324,(1989) 581;

J.L. Cardy and D.C. Lewellen, Phys. Lett. 259B (1991) 274. 
[15] A.B. Zamolodchikov, JETP Letters 46 (1987) 161; "Integrable field theory from conformal field theory" in Proceedings of the Taniguchi symposium (Kyoto 1989) and in Adv. Studies in Pure Math. 19 (1989) 641; R.A.L. preprint 89-001; Int. J. Mod. Phys. A4 (1989) 4235.

[16] A. Bilal and J.-L. Gervais, Phys. Lett. 206B (1988) 412; Nucl. Phys. B314 (1989) 646; Nucl. Phys. B318 (1989) 579; Nucl. Phys. B326 (1989) 222.

A. Bilal, Nucl. Phys. B330 (1990) 399; Int. J. Mod. Phys. A5 (1990) 1881.

T. Hollowood and P. Mansfield, Phys. Lett. 226B (1989) 73; Nucl. Phys. B330 (1990) 720 .

T. Eguchi and S.-K. Yang, Phys. Lett. 224B (1989) 373; Phys. Lett. 235B (1990) 282.

[17] D. Bernard and A. LeClair, Phys. Lett. 247B (1990) 309; Nucl. Phys. B340 (1990) 721; Commun. Math. Phys. 142 (1991) 99;

C. Ahn, D. Bernard, and A. LeClair, Nucl. Phys. B346 (1990) 409.

[18] V.S. Dotsenko and V.A. Fateev, Phys. Lett. 154B(1985) 291; Nucl. Phys. B251 (1985) 691;

J. Bagger, D. Nemeschansky and J.-B. Zuber, Phys. Lett. 216B (1989) 320;

S.D. Mathur, Nucl. Phys. B369 (1992) 433.

[19] P. Fendley, W. Lerche, S.D. Mathur and N.P. Warner, Nucl. Phys. B348 (1991) 66.

[20] P. Mathieu and M.A. Walton, Phys. Lett. 254B (1991) 106;

M.T. Grisaru, S. Penati and D. Zanon, Phys. Lett. 253B (1991) 357;

G.W. Delius, M.T. Grisaru, S. Penati and D. Zanon, Phys. Lett. 256B (1991) 164;

Nucl. Phys. B359 (1991) 125;

J. Evans and T.J. Hollowood Nucl. Phys. B352 (1991) 723; Nucl. Phys. B382 (1992) 662; Phys. Lett. 293B (1992) 100.

[21] N.P. Warner, " $N=2$ Supersymmetric Integrable Models and Topological Field Theories," in Trieste 1992, Proceedings, High Energy Physics and Cosmology, pp 143-179. Edited by E. Gava, K. Narain, S. Randjbar-Daemi, E. Sezgin, and Q. Shafi, World Scientific, 1993; ICTP Series in Theoretical Physics, v. 9.

[22] E. Martinec, Phys. Lett. 217B (1989) 431;

C. Vafa and N.P. Warner, Phys. Lett. 218B (1989) 51;

E. Martinec, "Criticality, catastrophes and compactifications," V.G. Knizhnik memorial volume, L. Brink et al. (editors): Physics and mathematics of strings.

[23] P. Fendley and K. Intriligator, Nucl. Phys. B372 (1992) 533; Nucl. Phys. B380 (1992) 265.

[24] A. LeClair, D. Nemeschansky and N.P. Warner, Nucl. Phys. B390 (1993) 653.

[25] A.B. Zamolodchikov and Al.B. Zamolodchikov, Ann. Phys. 120 (1979) 253.

[26] H. Saleur, S. Skorik and N.P. Warner, "The Boundary Sine-Gordon Theory: Classical and Semi-Classical Analysis," U.S.C. preprint USC-94-013, hep-th/9408004.

[27] P. Fendley, H. Saleur and N.P. Warner, Nucl. Phys. B430 (1994) 577. 
[28] E. Witten, Nucl. Phys. B185 (1981) 513; Nucl. Phys. B202 (1982) 253.

[29] S. Ghoshal, Int. J. Mod. Phys. A9 (1994) 4801; hep-th/9310188.

[30] H. Luckock, J. Phys. A24 (1991) L1057-L1064.

[31] C. Vafa, Mod. Phys. Lett. A6 (1991) 337.

[32] P. Fré, L. Girardello, A. Lerda and P. Soriani, Nucl. Phys. B387 (1992) 333.

[33] E. Witten, Int. J. Mod. Phys. A9 (1994) 4783.

[34] P.B. Wiegmann and A.M. Finkelstein, Sov. Phys. JETP 75 (1978) 204; 48 (1978) 1.

[35] P. Fendley, Phys. Rev. Lett. 71 (1993) 2485, cond-mat/9304031. 